\section{Abstract}

\title{
The impact of school reopening on COVID-19 dynamics in Bogotá, Colombia
}

\author{
Guido España1,\#, Zulma M. Cucunubá ${ }^{2,3, \#}$, Hernando Diaz ${ }^{4}$, \\ Sean Cavany ${ }^{1}$, Nelson Castañeda ${ }^{5}$, Laura Rodriguez ${ }^{6}$ \\ ${ }^{1}$ Department of Biological Sciences and Eck Institute for Global Health, \\ University of Notre Dame, USA \\ ${ }^{2} \mathrm{MRC}$ Centre for Global Infectious Disease Analysis, \\ J-IDA, Imperial College London, London, UK \\ ${ }^{3}$ Departamento de Epidemiología Clínica y Bioestadística, \\ Pontificia Universidad Javeriana, Bogotá, Colombia \\ ${ }^{4}$ Universidad Nacional de Colombia, Bogotá, Colombia \\ ${ }^{5}$ Escuela Tecnológica Instituto Técnico Central, Bogotá, Colombia \\ ${ }^{6}$ GCFEP-Universidad del Tolima, Ibagué, Colombia \\ \#Correspondence: guido.espana@nd.edu, \\ zulma.cucunuba@imperial.ac.uk,zulma.cucunuba@javeriana.edu.co
}

\begin{abstract}
The COVID-19 pandemic, caused by the SARS-CoV-2 virus, has affected millions of people around the world. In Colombia, 1.65 million cases and 43,495 deaths were reported in 2020. The exacerbation of poverty is a critical consequence of the pandemic, particularly in low- and middleincome countries. Schools have been closed in many places around the world to slow down the spread of SARS-CoV-2 and particularly in Latin America. In Bogotá, Colombia, public schools were closed since March 2020 and stayed closed for in-person instruction for the rest of the year, except for some schools that were open as a pilot for testing policies. To reconcile these two priorities in health and poverty, we estimated the impact of school reopening for in-person instruction in 2021. We used an agent-based model of SARS-CoV-2 transmission, considering social contact. The model includes schools that represent the set of private and public schools in terms of age, enrollment, location, and size. The model is calibrated to daily incidence of deaths in Bogotá. We simulated school reopening at different capacities, assuming high use of face mask, and evaluated the impact on the number of deaths in the city of different scenarios of school reopening by grade, and multidimensional poverty index. We found that school reopening, based on a correct use of face masks at $75 \%$ in $>8$ years of age, at $35 \%$ capacity had a small impact on the number of deaths reported in the city, assuming that overall mobility in the city was similar to the mobility during November. The increase in deaths was smallest when only pre-kinder was opened, and largest when secondary school was opened. Even at larger capacities, the impact
\end{abstract}


on the number of deaths of opening pre-kinder was below 10\%. Reopening other grades above $50 \%$ of capacity could significantly increase the number of deaths in the city. Reopening schools based on the multidimensional poverty index resulted in a similar increase in the number of deaths, independently on the level of poverty of schools. We conclude that the impact of schools reopening for in-person instruction is lower for pre-kinder grades and the magnitude of additional deaths associated with school reopening can be minimized by adjusting capacity in older grades.

\section{Introduction}

The COVID-19 pandemic has caused many deaths around the world and in Colombia. As of January 2021, more than 53 thousand COVID-19 deaths have been reported in Colombia. In Bogotá alone, more than 12 thousand people have died in the same period. Several interventions have been put in place to curb the spread of SARS-CoV-2, such as city-wide and partial lockdowns, mandatory use of face masks, contact tracing, and school closures [1]. Although interventions such as lockdowns are effective in reducing drastically and temporally the impact of COVID-19, they have negative impacts in society, especially in vulnerable communities $[2,3]$. In general, these closures disproportionately affect populations in lower socio-economic groups $[4,5,6]$. For instance, the ability of children to learn can be affected by school closures, since virtual learning requires guidance from parents. School closures can also increase the risk of harm by being out of school, such as domestic violence [7].

Schools are important for transmission of respiratory pathogens $[8,9]$, but the magnitude of its contribution to SARS-CoV-2 transmission is still unclear. School-aged children who are infected with SARS-CoV-2 have a lower chance of developing symptoms of COVID-19, and those who develop symptoms mostly experience milder clinical outcomes $[10,11,12]$. However, even if the risk of severe outcomes in children is lower, schools remain a potential source of transmission, which could have downstream effects in the community. In this regard, some limited evidence suggests that children under 10 years of age may be less susceptible to infection [13, 14, 15], but the evidence is not conclusive [13]. On the other hand, some studies suggest that children in secondary school could play a much more important role in transmission [16]. In fact, some studies suggest that secondary schools could have contributed to the spread of SARS-CoV-2 earlier in the pandemic $[14,17,18]$.

School reopening in the second semester of 2020 in various countries provided additional information about the impact of schools on COVID-19 dynamics. Some studies suggest that outbreaks within schools can be controlled, while others have shown some outbreaks linked to school. In Israel, large outbreaks were reported just 10 days after reopening [19]. In contrast, school reopening in England during summer 2020 showed that outbreaks in schools were uncommon but strongly related to the local incidence [20]. Similarly, the European CDC concluded that community transmission affected in-school incidence, but that school staff did not have a higher risk than other occupations [21]. In the United States, a study of 11 schools in North Carolina concluded through contact tracing that only 32 infections were acquired within schools and that adults were not infected by children [22]. A study in Mississippi suggest that attending in-person school or child care was not associated with increased risk of testing positive for SARS- 
CoV-2, but participating in social gatherings was [23]. However, given the lower probability of developing symptoms in children, it is difficult to assess the contribution of school reopening in specific communities. Hence, the risk of reopening schools should be evaluated in the local context.

Models are an important tool to understand the dynamics of infectious diseases and to plan public health interventions. Mathematical models have been used to estimate the potential burden of COVID-19 around the world [24, 25, 26]. For instance, in Chile, a stochastic mechanistic model has shown that early lockdowns were effective to reduce the impact of COVID-19 in Santiago de Chile, but they disproportionately benefited wealthier communities while penalizing vulnerable populations [2]. Within the context of school reopenings, various models suggest that the risk of reopening schools could be minimized with the use of interventions such as reduced class size, face-mask wearing, contact reduction by clustering students [27, 28, 29, 30, 31]. Importantly, these models agree that the risk of reopening is higher for older ages. In this study, we evaluate the impact of school reopening in the local context of Bogotá, Colombia, with the use of a stochastic agent-based model of COVID-19 dynamics calibrated to demographic, geographical, education characteristics, and epidemiological information of the city. We evaluated the impact of opening schools by grade and by the school-specific multidimensional poverty index, as well as of opening at different capacities on different dates.

\section{Results}

Our model captured the daily trends of deaths reported in Bogotá over time (Fig. 1A). In total, $75 \%$ of data points are captured by the predicted interval of the model. To capture the increase in transmission during the December holidays, an increase in community contacts of $61 \%$ was required (95\% CI: 60\%-65\%). The model captures the latest increase in deaths reported on January 14. Compared to the official reports of 126 deaths, the model estimates 103 (95\% CrI: 74-145). Our model captures trends of cumulative incidence of deaths and the age-stratified number of deaths by localities without including these data in the calibration (Figs. S2, S3). The model also reproduces trends in time and magnitude of the proportion of positive tests for PCR and antigen tests (Fig. S10). The estimated attack rate in the model was around $31.6 \%(95 \%$ CrI:31\%-31.8\%) by the first week of November in 2020 (Fig. 1B). Recent results from serological studies suggest that during the same period, 30\% (95\%CI: 27\%-33\%) of the population in Bogotá had been infected with SARS-CoV-2 [32]. Our calculations of the reproduction number $(\mathrm{R}(\mathrm{t}))$ suggest that early interventions (Fig. S1) decreased $\mathrm{R}(\mathrm{t})$ to around 1.0 in April (Fig. 1C). Estimates of the attack rate by age group showed that a larger proportion of younger adults (17-40) had been infected (approx. 40\% in November), in contrast to around $10 \%$ of people over 60 years (Fig. 1D). The attack rate also varied geographically with south-west areas showing higher attack rates (around 40\%) than north-east areas (10\%-20\%), consistent with the deaths reported in those localities (Figs. S2, S4).

Based on the assumptions adopted, our model projections show that in the event that schools reopened at full capacity and with no control measures at the end of January, an estimated total of 5356 deaths (95\% CrI:4951-5690) may result in the city from February up to August 31, 

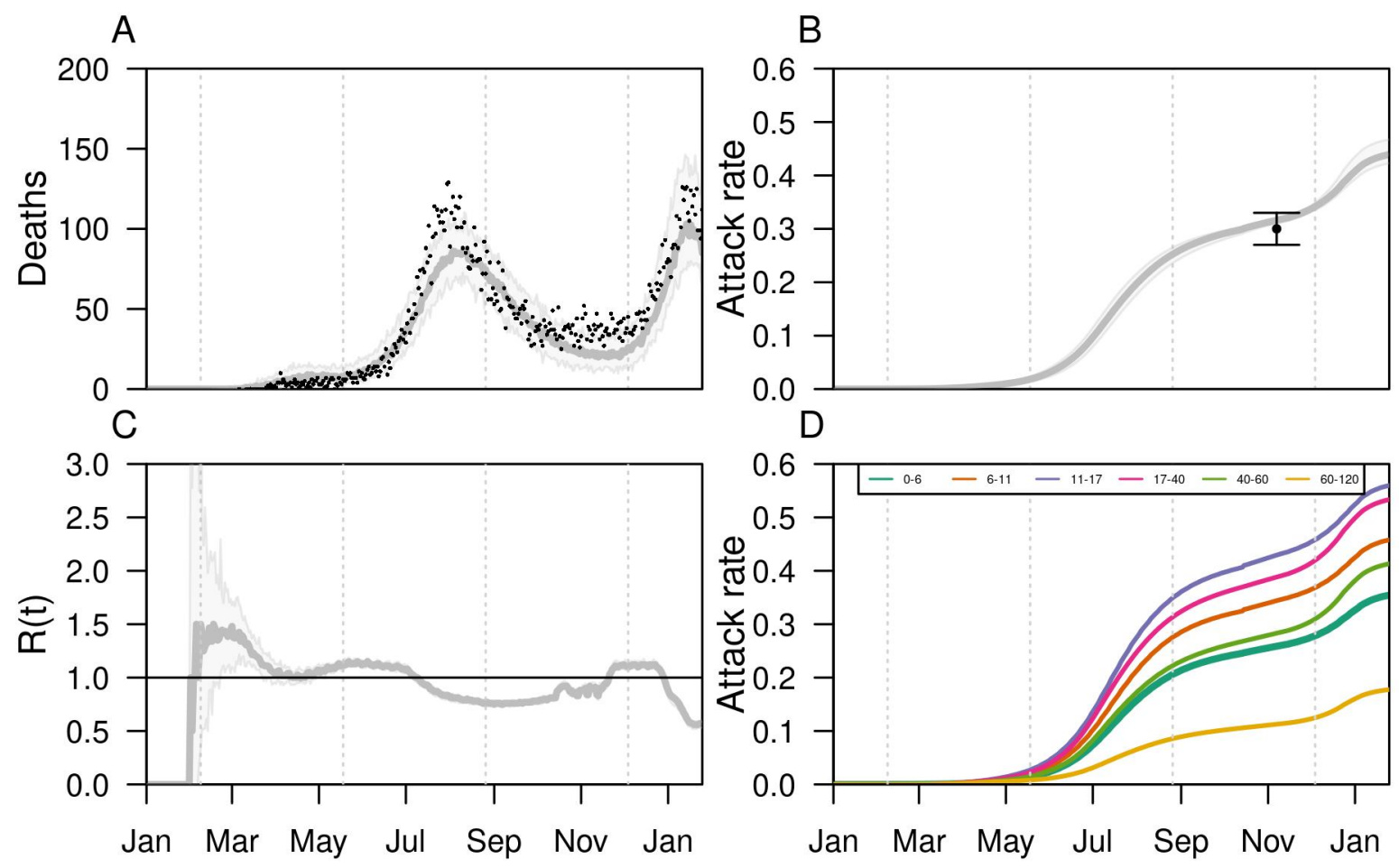

Figure 1. Model fit to data in Bogotá, Colombia. Assumption of lower (50\%) susceptibility in $<10$ years. A) Model fit to daily incidence of deaths. Black dots show the official data, and gray lines show the median estimate of the model with the $95 \%$ CrI represented by gray-shaded curves. B) Model estimates of attack rate in time represented by gray line (median) and shaded area $(95 \% \mathrm{CrI})$. The point and arrows show the median estimates and CI of official serological study in Bogotá. C) Estimated reproduction number in time. D) Estimated attack rate in time for different age groups.

2021. This number went up to 6252 (95\% CrI: 5836-6559) if universities were also opened in the same period with no control measures other than the use of face masks (Fig. 2B). In contrast, 1906 deaths (95\% CrI: 1779-2133) were estimated by the model simulations in the event that all schools remained closed (Fig. 2A). The impact of school reopening at full capacity resulted in a larger proportion of the population being infected in the simulation period with an attack rate of $60.0 \%$ (95\% CrI: 59-61\%), and 61\% (95\% CrI: $60.6-62.3 \%)$ if universities were open as well, compared to an attack rate of $43.2 \%$ (95\% CrI: 41.5-45.9\%) if schools remained closed (Fig. 2C). Our estimates of the reproduction number suggest a significant reduction in transmission early in 2020. However, the reproduction number increased to 2 in the scenario with schools fully opened (Fig. 2D).

The age of students attending in-person school affected the projected death toll of COVID-19 in the city. If only children under 6 years of age (pre-K) attended in-person schooling, a total 
of 1889 deaths were estimated (95\% CrI:1764-2188) at 35\% maximum capacity, which was a negligible difference from the baseline scenario of schools closed. Reopening pre-K grades at full capacity resulted in a small increase of $<200$ additional deaths in the whole city compared to the baseline scenario of all schools closed (Figs. 3A, 4, S11). Scenarios with older students attending in-person school impacted the total number of deaths at different levels depending on the operating capacity. For instance, if primary school reopened at $50 \%$, we did not see a large increase in the number of deaths (144 additional deaths). In contrast, in the model secondary school had to operate at a more restricted capacity of $35 \%$ to avoid increasing the number of deaths substantially in the city. At 50\%, more than 400 additional deaths were estimated. In fact, if secondary schools operated at $75 \%$ capacity, a large increase in the number of deaths was observed in the model projections with more than 1600 additional deaths in Bogotá in

- All closed $\quad-\begin{gathered}\text { School opening } \\ \text { at full capacity }\end{gathered} \quad-\begin{aligned} & \text { School + university opening } \\ & \text { at full capacity }\end{aligned}$ No interventions
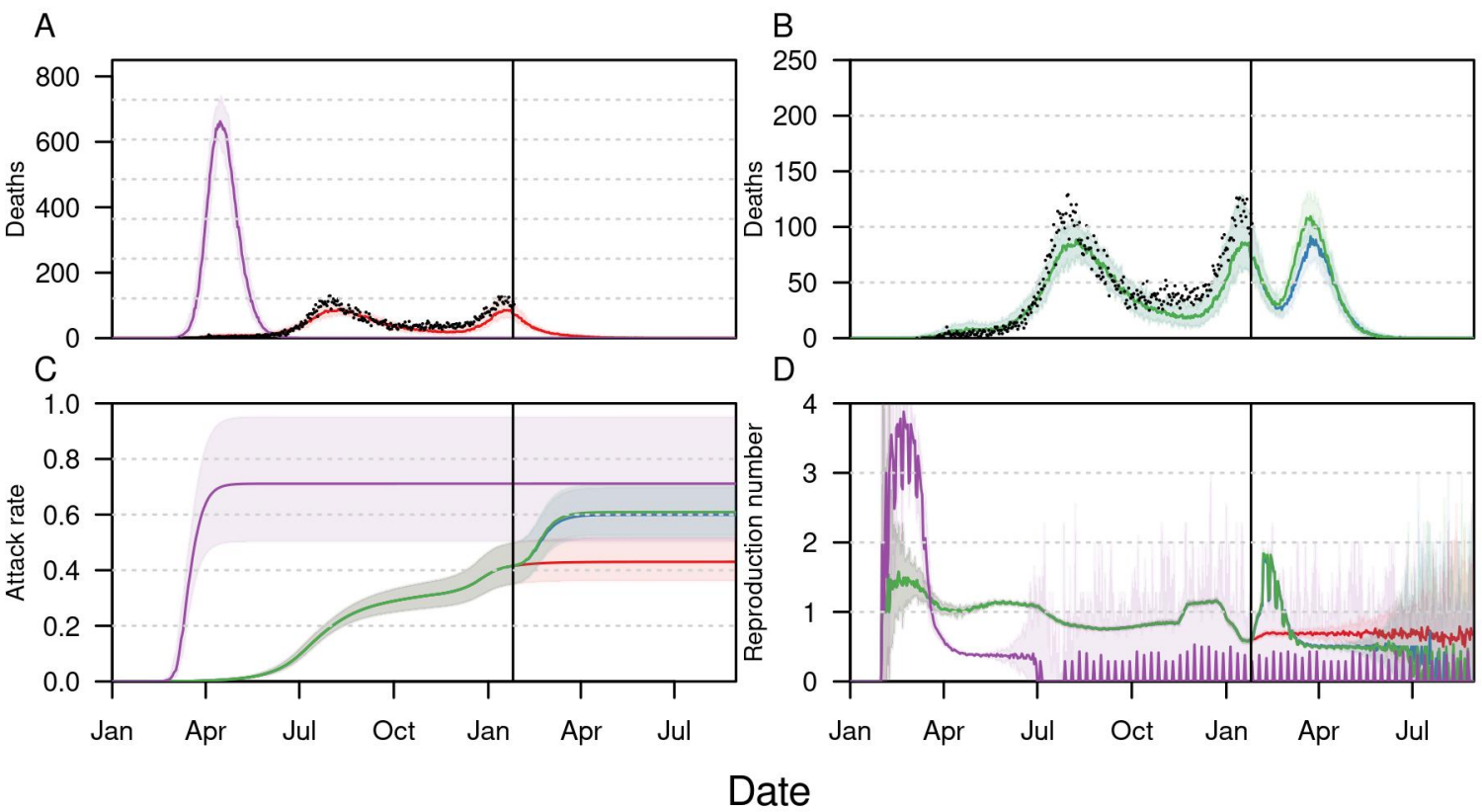

Figure 2. Projected impact of school reopening in Bogotá, Colombia. Assumption of lower (50\%) susceptibility in $<10$ years. A) Daily incidence of deaths for two extremes: a scenario in which there were no public health interventions (purple), and a scenario with the current public health interventions and assuming schools remain closed for the remaining of the simulation period. B) Daily incidence of deaths in two reopening scenarios: all K-12 schools reopen at full capacity but universities remain closed (blue), and all schools reopen, including universities. C) Estimated attack rate for the four scenarios considered. D) Estimated reproduction number for the four scenarios considered. All the scenarios were simulated until August 31, 2021. 
comparison to the closed scenario. The scenario in which students of all ages were able to attend in-person school at some capacity $(75 \%$ pre-K, $35 \%$ primary, $35 \%$ secondary) resulted in 431 additional deaths, compared to the closed scenario. At the same level of capacity in pre-K and secondary, but increasing primary capacity to $50 \%$, the number of additional deaths increased to 736 . Increasing primary capacity further to $75 \%$, increased the number of additional deaths to more than 1700 .

Across all scenarios, the dynamics in time showed that the magnitude of a third wave of infections could have a similar or greater magnitude than the previous two when schools opened at full capacity and no control measures were implemented (Fig. 4). Interestingly, assuming current levels of testing capacity, the positivity of PCR showed an association with the magnitude of future outbreaks (Fig. S11). In general, levels under 10\% suggested a low impact on the citywide health care system, whereas levels of at $15 \%$ or above suggested a third wave of large enough magnitude that could put the health system under pressure (Fig. S11).

We also evaluated an alternative scenario in which people of all ages had the same susceptibility to SARS-CoV-2 infection. The model calibration resulted in similar patterns but with higher attack rate in lower ages (Fig. S9D). Qualitatively, the impact of reopening strategies based on income and grades remained similar to our main assumption of susceptibility (Fig. 3B, S5B). Grades of younger ages had a lower impact on the burden of COVID-19, compared to older ages. Numerically, the impact of school reopening was slightly higher under this assumption. For instance, if all schools and universities reopened at full capacity, around 5048 additional deaths were estimated, compared to 4346 additional deaths caused in the baseline assumption of children $<10$ being less susceptible. Similarly, if all schools were reopened at full capacity, 4030 additional deaths were estimated with the model, in comparison to 3450 additional deaths with the baseline assumption of susceptibility. In addition, reopening schools on different dates under this alternative assumption of susceptibility resulted in temporary reduction in the number of maximum daily deaths (Fig. S12). Another assumption in the model was that asymptomatic infections had the same ability to transmit the virus. We relaxed this assumption and evaluated the impact of lower infectiousness of asymptomatics as $75 \%$ of the infectiousness of symptomatic individuals[33]. Because of the higher rate of asymptomatics in children, a lower infectiousness of asymptomatics resulted in a lower impact of school reopening (Fig. S6). Compared to the baseline assumption of infectiousness, at $75 \%$ pre-K capacity, $50 \%$ primary, and $35 \%$ secondary, 2265 total deaths (95\%CI: 1989-2910) were estimated. This estimate represented 377 fewer deaths than the same scenario under the baseline assumption of infectiousness (2642 95\%CI:2448-2850).

Policies of reopening based on the multidimensional poverty index of schools (MPI, high MPI = high poverty in schools) did not show an appreciable difference in the number of deaths (Figs. S5A, S8). In the event that only the schools classified the lowest MPI value were to open for all school grades at 35\% capacity, a total of 38 additional deaths were estimated, in comparison to the scenario in which schools remain closed. We found that at $35 \%$ capacity, schools with the highest MPI resulted in 58 additional deaths. At full capacity, scenarios of reopening schools with the lowest MPI(Q0-Q1) resulted in around 1529 additional deaths in the whole city, compared to 1300 additional deaths in the whole city when schools with the highest MPI (Q3-Q4) reopened. Overall, reopening schools with the highest MPI had a smaller impact 
on the number of deaths, but differences among schools were small. These results contrast with the impact of COVID-19 being much higher in lower income areas in the south-west of the city (Fig. S4). At full capacity, these areas might be more resilient to school reopening given the large proportion already infected in those areas.

In the event that school reopening increased the mobility to baseline levels, our results suggest an increase in the impact of reopening at any level (Fig. 3, S5). The increase was uniform across all scenarios considered. For instance, reopening pre-K grades increased the number of deaths from 1889 to 2287 at $35 \%$ capacity, while pre-K (75\%) + primary $(35 \%)+$ secondary (35\%) increased from 2337 to 3008 deaths. Similar increments were observed for the scenarios of reopening by socioeconomic status. In general, all the scenarios increased the tally of deaths in the event of increased mobility, including the scenario where schools remained closed (from 1906 to 2292 deaths), which indicates that the level of community contact while schools are open affect the capacity of schools to remain open without increasing the burden of COVID-19 in the city.

Delaying the date of school reopening reduced the peak of the number of deaths projected within the simulation period for scenarios of high capacity but had a negligible effect on scenarios of low capacity (Fig. 5A,D). At full capacity, our projections suggest that reopening on January 25 would have a higher peak of deaths (90 per day) than delaying school reopening to February 25 (78 deaths per day) and March 25 (73 deaths per day). Similar differences were observed at $75 \%$ capacity with the highest number of deaths per day (55 deaths per day) reported in the baseline scenario of reopening in January 25, 2021, followed by 48 deaths reopening delayed 1 and 2 months (Fig. 5B,E). In contrast to the full capacity scenario, at $35 \%$ the model projections showed that schools alone would not produce a significant increase in the overall number of deaths or the proportion of people infected (Fig. 5C,F). Although delaying school reopening had an impact in the maximum number of daily deaths, the final percentage of people infected was around $60 \%$ for all three dates (Fig. 5D), suggesting that the cumulative contribution of school reopening remained the same.

\section{Discussion}

We modeled school reopening using a detailed agent-based model calibrated to the local context of Bogotá, Colombia. Our model was calibrated to local incidence of deaths and validated to data excluded from calibration, such as the heterogeneous spread of disease across the city and data from a serological study. A strength of our model is that it includes a detailed description of individuals in the city and their changes in behavior to respond to public health measures. Interestingly, our results show that restrictions in mobility and interactions have had an impact in reducing the impact of COVID-19 in the health system. Under an assumption of no public health interventions, we observed a large outbreak with a peak of around 600 deaths per day, and an attack rate close to $75 \%$, which was similar to attack rates observed in unmitigated outbreaks in South America [34]. In addition, calibrating the model to the local context with a detailed description of the school population, enabled us to determine the impact of school reopening at different age and socio-economic levels. Our model projections suggest that school reopening 
may lead to a substantial increase in SARS-CoV-2 transmission in Bogotá, Colombia, but this effect can be mitigated by managing the school capacities and increasing control measures. Our results were consistent with other modeling studies suggesting that younger grades could have a lower impact in transmission than older grades [27, 28, 35].

Our results suggest that reopening schools for in-person instruction at full capacity for all grades could result in an increase of deaths of similar or greater magnitude to the previous two outbreaks reported in July and December of 2020. However, the impact on city-wide transmission

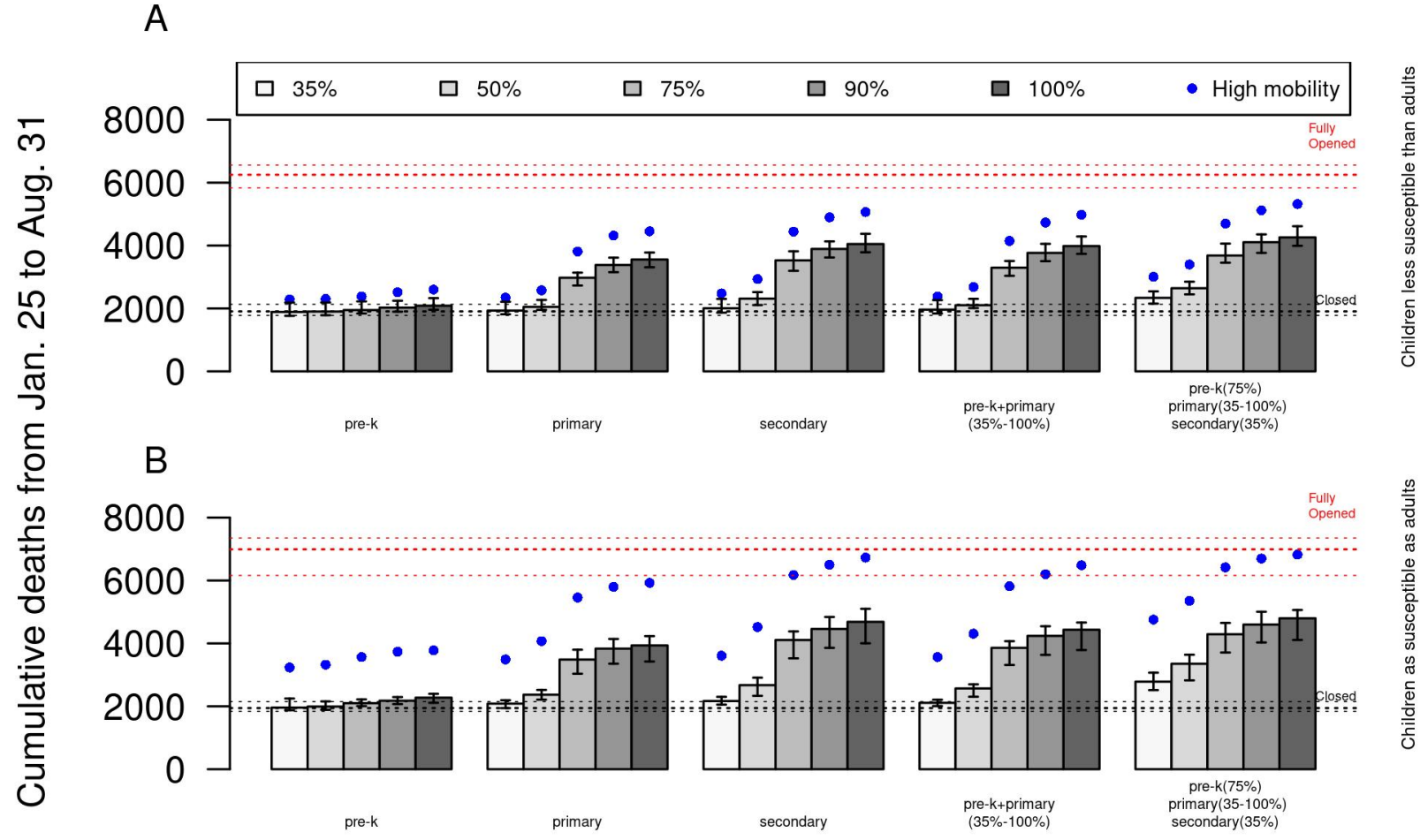

Figure 3. Total cumulative deaths under different school reopening strategies from January 25 to August 31, 2021. A) Cumulative deaths of scenarios in which schools reopen by grades with an assumption of lower (50\%) susceptibility in $<10$ years. B) Cumulative deaths of scenarios in which schools reopen by grades with an assumption of equal susceptibility for all ages. From left to right, the first group of bars show exclusive reopening by grade groups in which the other grades remain closed. The fourth group of bars (prek+primary) represents a scenario in which prek primary and primary reopen at different capacities but secondary remains closed. The last group shows a scenario in which all grades go to in-person school at some level, with prek fixed at $75 \%$, secondary fixed at $35 \%$, and primary varying from $35 \%$ to $100 \%$. Blue dots show the median estimate of the same scenario with higher mobility in the city when schools reopen. In all scenarios, we assumed long-term protection after SARS-CoV-2 infection. 
was much lower for scenarios of schools operating at reduced capacity for in-person instruction, and assuming correct face-mask use at $75 \%$ in children of 8 years of age and older. Particularly, the model showed that reopening prekinder, even at almost full capacity, may not lead to a substantial increase in the overall deaths in the city. This lower risk for younger grades is the result of a combination of factors such as lower susceptibility, the total number of students, and the limited contacts of younger children outside of school. This impact remained similar even in when we assumed the same susceptibility to infection by younger children compared to adults.

The model reproduces well transmision patterns within schools, which support previous findings by Mc Gee et al. [36], where they illustrate transmission patterns of children in primary having more contacts within the same grade and children in secondary having more contacts outside their grades. In our model, younger children represent a lower impact in COVID-19 mortality in Bogotá due to three determining factors: i) the size of the schooling population increases with age, ii) in schools the mixing contact patterns of children increases with age, iii) the risk of death increases with age. These trends remained similar regardless of the assumption on susceptibility to infection for which we modeled similar or lower susceptibility in children.

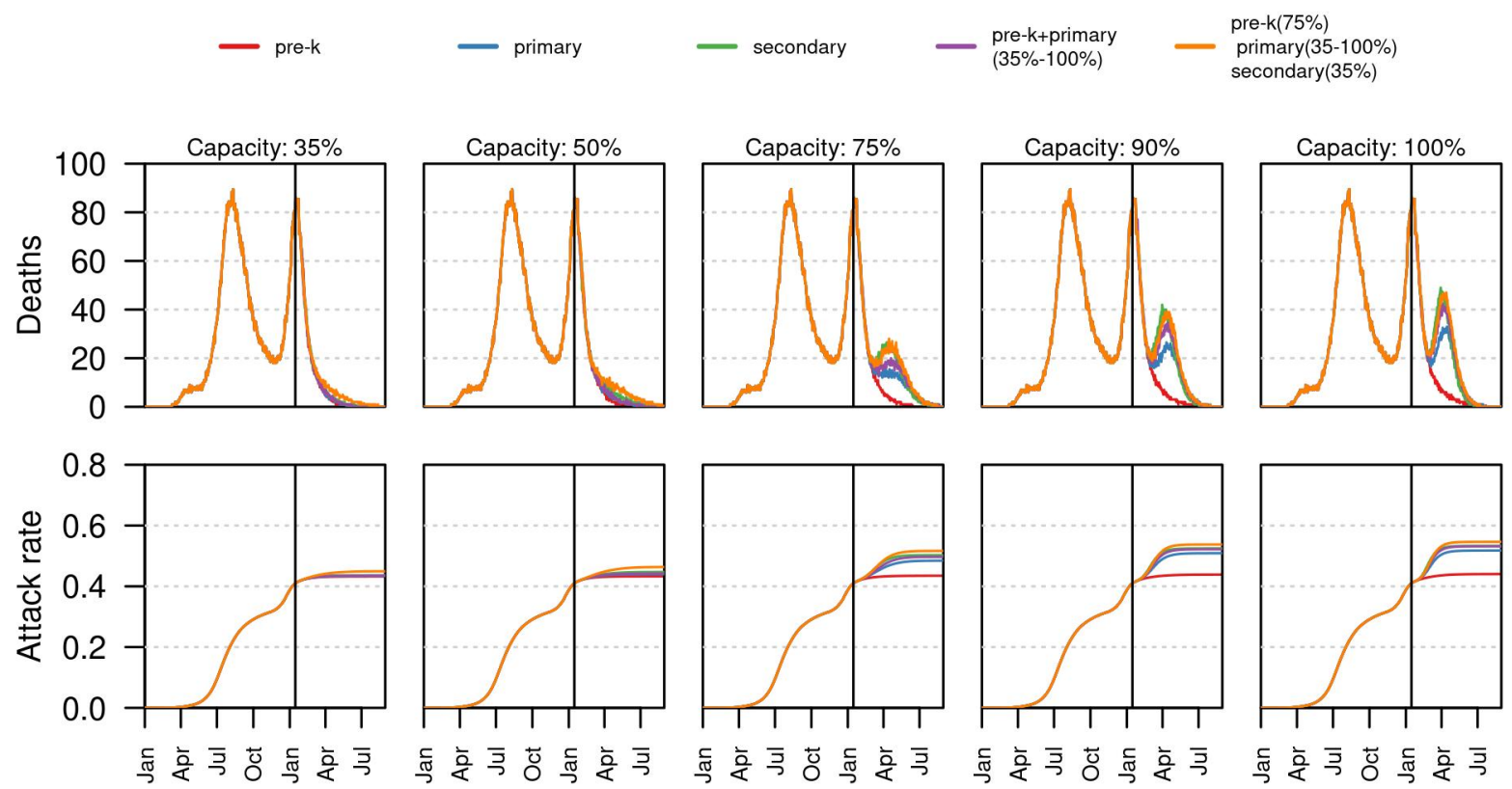

Figure 4. The impact of school reopening strategies in time. Each column shows a different capacity level. Top panel shows the median daily incidence of deaths for each reopening strategy based on grades. Bottom panel shows the estimated attack rate for each of the reopening scenarios. Vertical black line shows the timing of school reopening (January 25, 2021). All scenarios were simulated up to August 31, 2021. Assumption of lower (50\%) susceptibility in $<10$ years. 
Another interesting finding of our model is that PCR positivity in the whole city showed a relationship with actual transmission and may help monitoring the safety of school reopening scenarios. Based on current testing capacity, and after the second wave, at less than $10 \%$ of PCR positivity, our results suggest that it may be safe for school reopening with minimal impact in the total number of deaths. Whereas, levels of $10-15 \%$ could be indicative of a moderate third wave, and levels greater than $15 \%$ could indicate a third wave large enough to put the health system under potential high pressure.

An important finding is that the level of mixing needed in the model to reproduce the peak in December related to christmas and new eve holidays was greater than any other over the year. These high mixing rates in the community (household to household and family visits) over December resulted in a large and rapid second peak. Similar patterns may be observed over other holidays such as Easter break, but we have not included that assumption in our model. Importantly, any increase in mobility trends leads in the model to increase in community mixing

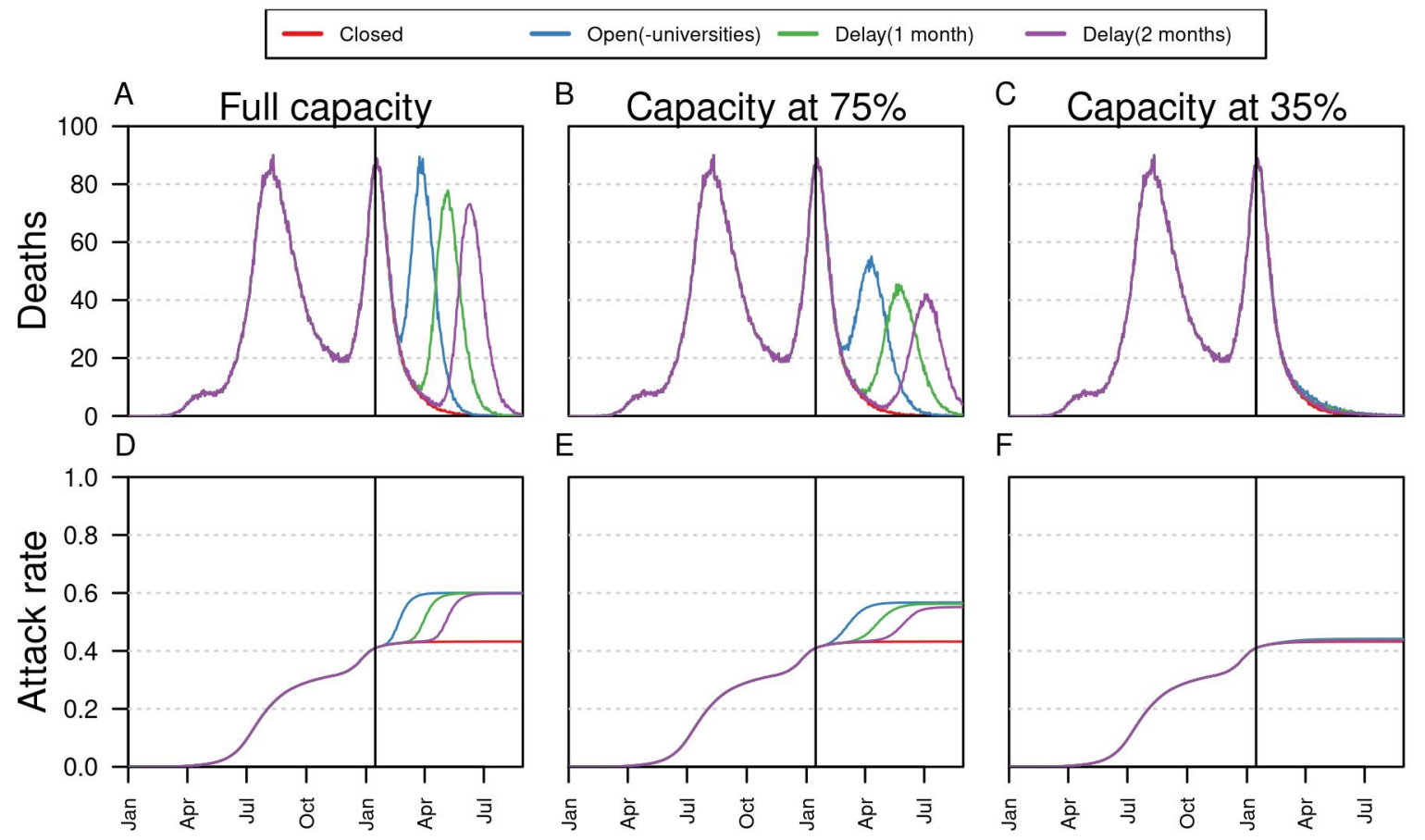

Figure 5. The impact of delaying school reopening. Each column shows a different capacity level. Red lines represent a scenario in which all schools remain closed, blue lines represent K-12 schools open, green and purple lines show scenarios of delaying school reopening by 1 and 2 months, respectively. Top panel shows the median estimate of daily incidence of deaths. Bottom panel shows the median estimate of attack rates for each scenario. Vertical black line shows the initial date of school reopening (January 25, 2021). All scenarios were simulated up to August 31,2021 . Assumption of lower $(50 \%)$ susceptibility in $<10$ years. 
which in turn may result in increased transmission. A third wave in Bogotá, related to school opening is related to both, an increase in mixing patterns within schools and an accompanying increase in community transmission outside school.

The burden of COVID-19 has been heterogeneous across parts of the city, with a larger impact in neighborhoods of lower socioeconomic status. This is not unique to Bogotá. Studies have shown that vulnerable communities are less able to comply with public health interventions that reduce mobility, increasing the burden of COVID-19 in such communities [2]. Our data-driven approach allowed the model to reproduce this geographic heterogeneity. Nonetheless, our school reopening scenarios based on their MPI showed a small difference in the impact of schools across socioeconomic status. The MPI of each school measures the level of poverty of schools attending the school, which means that schools with higher MPI have a higher proportion of students under poverty conditions. These students live in areas that have had a higher burden of COVID-19, increasing their probability of being already exposed to the virus. Another factor associated with the small difference across MPI values is that schools attract few students from various neighborhoods around the city, increasing the probability that schools located in neighborhoods with low transmission could have imported infections from neighborhoods with high transmission.

Various assumptions were made in our model. Importantly, we assumed that children under 10 years of age are $50 \%$ less susceptible than older ages[15]. However more studies would be needed to determine whether children are in fact less susceptible than adults [13]. Consequently, we also evaluated the impact of school reopening under the assumption of equal susceptibility for children and adults. Even under this assumption, younger grades consistently had a lower impact on transmission than older ones. Although, the overall impact of school reopening was slightly higher under the assumption of equal susceptibility. We also assumed that children are able to transmit SARS-CoV-2 at the same level as adults. Although children are less symptomatic than adults [11], published studies suggest that children could be as infectious as adults [13, 37, 38]. We also evaluated a scenario in which relative to symptomatic infections, asymptomatic infectiousness was slightly lower (75\%). Under this assumption there was a reduced impact of school reopening. This reduction was proportionally larger for scenarios of low or moderate capacity, but at higher capacities the reduction was lower. The ability of children to transmit the virus emphasizes the importance of face-mask adherence, maintaining physical distancing in schools, and other interventions, such as controlling capacities in schools.

Another assumption made in the model is that levels of mobility would increase up to levels seen in November, 2020. However, the model does not include adaptive behaviors such as parents changing schedules in the case that their kids attend in-person school, could have an impact on mobility and contacts across the city. Mobility could also increase by students using public transportation to go to school, which was not included in the model. Hence, mobility could increase even more than levels seen in November 2020. Consequently, we assumed a scenario with higher mobility up to baseline pre-pandemic levels. At this level of mobility, deaths increased slightly and uniformly across all scenarios studied. Although we are unable to project the full extent of future mobility and levels of contacts within the city, this result highlights the importance of continuing control measures in the city to maintain acceptable levels of transmission when schools reopen. 
We considered a reduced set of possible reopening strategies to focus on quantifying the impact of school capacity by age and socioeconomic status. Another strategic aspect not considered is the effect of face-mask adherence within school, which has been explored in similar analyses of school reopening[30]. Instead, we set the baseline level of face-mask adherence to $75 \%$, based on city surveys. Furthermore, we did not consider reactive interventions to control the spread of SARS-CoV-2 within schools, such as contact tracing, classroom closures, or individual school closures. Another simplification of the school reopening strategy is that we simulated uniform mandates and compliance with public health measures across the city. The reality is that some schools would be able to enforce interventions more than others. Nonetheless, our simulations represent an average of the city-wide reopening strategy. In general, our results highlight the importance of controlling school capacity at different levels depending on the school grades.

School closures might have contributed to reducing the impact of COVID-19 during the early months of the pandemic, and may continue to do so [17]. However, the risks of reopening schools should be balanced with the negative outcomes of long-term school closures. Our model shows that with reduced capacity and control measures in place, the city-wide impact of school reopening could be greatly reduced; although it would nevertheless lead to additional deaths in all but the most stringent of cases. In contrast, high capacities and low control measures in place could result in large outbreaks, and large increases in deaths, as a consequence of in-school transmission chains extending to the rest of the city[27].

\section{Limitations}

The model is a representation of the city including high resolution demographic and geographical data. However, the model is unable to reproduce the full range of heterogeneities in the school system. For instance, we assumed classes are undertaken in classrooms and not outdoors. This could ignore potential benefits of schools with the capacity to set up outdoor classrooms. Similarly, the model simplifies school structures across socioeconomic status, which in reality may have different characteristics. In addition, the reopening strategies evaluated in this study does not include reactive measures that schools could take to reduce the impact of outbreaks once they are identified. This could mean that our estimates could underestimate the impact of school reopening in some aspects and overestimate it in others. Although in the school opening scenarios we have assumed the current mobility levels will increase up to November levels and a scenario of high mobility with baseline levels of mobility, the model is unable to predict the levels of contacts outside schools increased for other reasons and what would be the impact on intra-school transmission. The model does not explicitly include the potential impact of public transportation or school's transportation. Finally, our model does not include potential impact of waning immunity or other variants with increased transmission or immunity escape capacities, and does not include potential vaccination scenarios. 


\section{Methods}

\section{Data}

Demographic data was obtained from IPUMS-International, and the city planning secretary of Bogotá [39, 40]. Demographic data on long-term care facilities were obtained from the Census and the ministry of health [41,42]. We manually geo-located these institutions using google maps.

Information about the number of schools, their capacity by age, and geo-location were obtained from the city's Secretary of Education, which also provided us with a list of the Multidimensional Poverty Index (MPI) for each school. The MPI of each school represented the level of poverty of its students, not the location of the school. For institutions of superior education, we obtained a list with capacities from the national Ministry of Education [43] and manually geolocated them using google maps. We obtained data-sets for workplaces, including the number of workers and geo-location of each formal and informal workplace in the city, from the Secretary of Education.

We used publicly available data to approximate trends in the adoption of public-health interventions, such as lockdowns and the use of face masks. For lockdowns, we used the Google Mobility Reports [44] on the time-varying proportional change of people staying at home since March, 2020. We later adjusted the magnitude of this time-series to fit the model. To approximate the geographical variation of lockdown compliance, we combined the time-varying trends from Google Mobility Reports with data from the Grandata project[45], which includes changes in mobility by day at the census-tract level (Unidad Catastral) but were not as frequently updated as the reports from Google. The adoption of face masks was approximated using data from google trends on the specific search terms 'tapabocas' and 'mascarilla' from February until October, 2020[46]. Assuming that people who bought masks would subsequently wear them, we computed the cumulative interest in those terms and used a scaling factor in the calibration step to estimate the proportion of people wearing face masks over time.

We used daily incidence data on deaths from the surveillance system of the National Institute of Health (INS) [47]. We also used data stratified by age and locality in Bogotá from the city's Secretary of Health, to validate the model performance. Serological studies were also used to compare model performance [32].

\section{Description of agent-based model}

We modeled the dynamics of SARS-CoV-2 transmission with an agent-based model using a modified version of the platform FRED[48], which was originally developed to simulate influenza pandemics at the University of Pittsburgh. This version of the model has been described elsewhere [30]. This model has also been used previously to simulate COVID-19 dynamics in school reopening in Indiana [30] and to forecast the weekly incidence of death in seven states in the United States as well as to study the impact of non-pharmaceutical interventions [49, 50]. In our model, each inhabitant of Bogotá is modeled as an agent who has a set of daily activities, such as school attendance or commuting to work. Transmission of the pathogen can occur when an 
infectious person visits the same place a susceptible person visited the same day. We assumed that proportion of the overall infectious people in the city would visit long-term care facilities, potentially infecting their residents. Finally, the probability of transmission partly depends on the number of effective contacts that a person has for each location type. These numbers of contacts were assumed to be those previously calibrated values to influenza for each location type [48].

Transmission and disease progression is based on a modified SEIR model. Latency and infectious periods were drawn from distribution calibrated to the average generation interval in Singapore [51]. The probability of developing symptoms increased with age [10]. Similarly, the probability of death increases with the age [52]. We assume that agents who recover from infection acquire long-term immunity. We assumed children and adults have the same capacity to transmit the virus to others upon exposure, although they were less likely to develop symptoms. We assumed that asymptomatic and symptomatic infectious individuals had a similar probability of infecting a susceptible agent upon exposure, but relaxed this assumption in an alternative analysis in which asymptomatic infectiousness was set to $75 \%$ that of symptomatic infections [33]. Based on limited evidence on children susceptibility, we assumed two possibilities i) that children under 10 years of age were $50 \%$ less susceptible to infection compared to older children and adults ii) that children have the same susceptibility to infection as adults [14].

Non-pharmaceutical interventions were incorporated in the model to modify agents' behavior to curb the burden of COVID-19. We simulated lockdowns by restricting agents' mobility to their household and local community based on daily reports of human mobility in the city[44]. The effect of people wearing face masks was included in the model by reducing the probability of transmission of an susceptible individual upon exposure. The efficacy of this measure was determined as the lower bound of the odds ratio from estimates of SARS-CoV efficacy in nonhealth care settings (aOR: 0.73)[53]. The temporal trends of people wearing face masks was adjusted from google trends on specific search of face masks in Bogotá ('tapabocas', 'mascarilla') [46]. The proportion of people wearing face masks depended on the specific location and the age of the agent. Only people older than 7 were eligible to wear a face mask. For workplace and community, temporal trends from google trends were adjusted with a scaling factor in the calibration step. We assumed that people did not wear face masks in their households. In the event that schools reopen, we assumed that $75 \%$ of students older than 7 years of age would properly wear face masks.

The model includes schools that represent the set of private and public schools in Bogotá in terms of age, enrollment, location, and size. Transmission of the virus in schools can occur because of contacts inside the classroom or with the rest of the school [48]. We assumed that for a person in the school, the number of contacts in the classroom is double the number of contacts with the rest of the school. The size of each classroom was determined by age in agreement with the average size by grade in the city schools. The model also includes the population of teachers.

\section{Synthetic population}

We created a synthetic population that matches geographical and demographic characteristics of the population in Bogotá. We used publicly available micro-data from the IPUMS-International 
database [39] and used an iterative proportional fitting algorithm using the simPop package in $\mathrm{R}$ to fit age, household-composition, and population size by each census tract unit (Unidad Catastral) [54]. We also included long-term care facilities in the model based on data from the ministry of health. The synthetic population was fit to census-tract data and it also represents the city-wide population by age and household population (Fig. S7A,B). The geographical density Bogotá is distributed in neighborhoods and localities, which contain several neighborhoods. The population density by census tract is shown in figure S7C. Also, the precise location of households, schools, and workplaces is shown in figure S7D. We focused on the urban localities and omitted the locality of Usme, which is mainly rural.

In the synthetic population, students in prek, primary, and secondary school were assigned to school based on data from the Secretary of Education for each grade. Students were assigned to a school in three sequential steps. First, for each student, a list of schools with availability for the student's age was created. Then, we used data from the Secretary of Education to determine a matrix of locality of residence vs locality of school. Based on this matrix, we selected a locality to assign the student's school. Third, we assigned the school of the student based on two criteria, if the locality is the same as the student's household, we assign the student to the closest school with availability, if the locality is not the student's household locality, we assigned the school at random within that locality. For students in higher education, such as universities, we obtained a list of institutions with their student capacity from the Ministry of Education [43]. We randomly assigned students in higher education institutions based on their capacity.

Workers were assigned to workplaces based on a data set of formal and informal workplaces. This database included the number of workers and geo-location of the workplace. We used a mobility survey in Bogotá to create a matrix of locality of household vs locality of workplace. Based on this matrix, we assigned workers to workplaces based on distance and capacity.

\section{Model initialization and calibration}

To reproduce the timing of SARS-CoV-2 importation in Bogotá, we initialized the model based on international and domestic importations in the city using case fatality risk and locally reported death data. Detailed description of these methods are described elsewhere [55, 30]. We fitted a GAM to the mobility trends from the percentage change on mobility for places of residence, and assumed that future mobility would increase up to values observed in November, 2020. We defined the maximum mobility in the city as $0 \%$ of people sheltering in place and the minimum mobility in the reports as $100 \%$ of people sheltering in place. Then, we scaled these trends based on a scaling factor that we calibrate. We adjusted the numerical values of six model parameters to reproduce the daily incidence of deaths in Bogotá. Namely, the scaling factor for imported infections, a scaling factor for importation of infections to long-term care facilities, the probability of transmission upon exposure, the adherence with shelter-in-place and face-mask recommendations, and a percentage increase of community contact during the holidays. We calculated the likelihood of the model given the observed daily incidence of deaths for 2,000 simulations of the model with combinations of these parameters, $\vec{\theta}$, using a sobol design sampling algorithm with the sobolDesign function in $\mathrm{R}[56,57]$. We then sampled from these 2,000 parameter sets based on their likelihood, which was calculated as $\mathcal{L}\left(\vec{\theta} \mid D_{t}\right)=\operatorname{Negative} \operatorname{Binomial}(r, p)$, where $D_{t}$ is the 
daily incidence of death on day $t$ and $r$ and $p$ are size and probability parameters, respectively. We informed $r$ and $p$ using the conjugate prior relationship between a beta prior and negative binomial likelihood.

We validated the model with data excluded from the calibration process. Serological studies were carried out in Bogotá between October 26th and Novebmer 17th, 2020 to estimate the proportion of the population infected with SARS-CoV-2 [32]. We estimated daily attack rate in our model and compared the values to the serological study.

We also contrasted our model to the daily positive rate of PCR and antigen tests. We assumed perfect specificity and sensitivity of 0.85 for PCR [58] and 0.75 for antigen tests [59]. The proportion of positive tests were calculated as

$$
P(P \mid T)=\operatorname{sensitivity}(P(C \mid T)+P(I \mid T))+(1-\text { specificity }) P(U \mid T),
$$

where $\mathrm{T}$ refers to $\mathrm{PCR}$ or antigen tests administered, $\mathrm{C}$ to symptomatic infections, I to asymptomatic or pre-symptomatic infections, and $U$ to uninfected individuals. As explained elsewhere [30], $\mathrm{P}(\mathrm{C} \mid \mathrm{T})$ can be expressed as

$$
P(C \mid T)=\frac{P(C)}{P(C)+r(1-P(C))},
$$

where $r=P(T \mid \neg C) / P(T \mid C)$. $\mathrm{P}(\mathrm{I})$ and $\mathrm{P}(\mathrm{U})$ can be written as

$$
P(I \mid T)=\frac{r P(I)}{P(C)+r(1-P(C))}
$$

and

$$
P(U \mid T)=\frac{r P(U)}{P(C)+r(1-P(C))} .
$$

\section{School reopening scenarios}

To evaluate the impact of different variables for school reopening, we focused on strategies of exclusive reopening by each variable. These variables included grades, multidimensional poverty index, and geographical location of schools. We simulated reopening strategies of grades including, prek, primary, and secondary school. We modeled varying degrees of school capacity by modulating the probability of a student to go to school in a specific day. In the model, reduced capacity does not imply greater physical distancing within schools. We varied the capacity of reopening for in-person students from $35 \%$ to $100 \%$ for each set of grades. We also evaluated the impact of reopening prek and primary schools together at similar capacity levels from $35 \%$ to $100 \%$. Finally, we simulated a scenario in which students from all ages were able to attend inperson school at some level with $100 \%$ prek, $50 \%$ primary school, and secondary school capacity varying from $35 \%$ to $100 \%$.

The multidimensional poverty index (MPI) is an international measure of poverty that includes monetary poverty metrics and other acute deprivations in health and living standards 
[60]. We used an adjusted MPI for each school, which represents the overall intensity of poverty in the school's students. Then, we sorted schools based on their MPI and student population size, and grouped the schools based on their population quartile in four groups (MPI Q0-Q1, Q1-Q2, Q3-Q4) from lower to higher MPI index. To estimate the effect of MPI of schools in school reopening, we simulated exclusive reopening for each of the four determined groups.

We also simulated extreme scenarios of school reopening in which schools remain at their current level of attendance or they are open at full capacity. Finally, we evaluated the impact of delaying school reopening by 1 or 2 months from the initially planned reopening (January 25, 2021). In all simulations, we evaluated the impact of reopening schools as the total number of deaths reported in the city from January 25 to August 31, 2021.

\section{Acknowledgements}

We thank the University of Notre Dame Center for Research Computing for computing resources. GE received funding from an NSF RAPID grant (DEB 2027718). HD received partial funding from the National University of Colombia (Universidad Nacional de Colombia (HERMES 50419)). ZMC receives funding from Medical Research Council (MR/R024855/1). ZMC holds an honorary role (without payment) in the Scientific Advisory Group on epidemiological modelling at Secretaría de Salud in Bogota.

The authors thank the Secretary of Education of Bogotá for providing us with data about schools, and the Secretary of Health of Bogotá for providing us with epidemiological data. We thank Roberto Angulo for his guidance on planning and interpreting results related to poverty.

\section{References}

[1] Alcaldía Mayor de Bogotá. DECRETO No. 121. Apr. 2020.

[2] Nicolò Gozzi et al. "Estimating the Effect of Social Inequalities in the Mitigation of COVID19 across Communities in Santiago de Chile". In: medRxiv (2020). DOI: 10.1101/2020.10. 08.20204750. eprint: https://www.medrxiv.org/content/early/2020/10/13/2020.10.08. 20204750.full.pdf.

[3] Vida Abedi et al. "Racial, Economic, and Health Inequality and COVID-19 Infection in the United States". In: Journal of Racial and Ethnic Health Disparities (Sept. 2020). ISSN: 2196-8837. DOI: 10.1007/s40615-020-00833-4.

[4] Mads Meier Jæger and Ea Hoppe Blaabæk. "Inequality in Learning Opportunities during Covid-19: Evidence from Library Takeout". In: Research in Social Stratification and Mobility 68 (2020), p. 100524. ISSN: 0276-5624. DOI: 10.1016/j.rssm.2020.100524.

[5] Jörg M. Fegert et al. "Challenges and Burden of the Coronavirus 2019 (COVID-19) Pandemic for Child and Adolescent Mental Health: A Narrative Review to Highlight Clinical and Research Needs in the Acute Phase and the Long Return to Normality". In: Child and Adolescent Psychiatry and Mental Health 14.1 (May 2020), p. 20. ISSN: 1753-2000. DOI: 10.1186/s13034-020-00329-3. 
[6] Elisabeth Beaunoyer, Sophie Dupéré, and Matthieu J. Guitton. "COVID-19 and Digital Inequalities: Reciprocal Impacts and Mitigation Strategies". In: Computers in Human Behavior 111 (2020), p. 106424. ISSN: 0747-5632. DOI: 10.1016/j.chb.2020.106424.

[7] Megan L. Evans, Margo Lindauer, and Maureen E. Farrell. "A Pandemic within a Pandemic - Intimate Partner Violence during Covid-19". In: New England Journal of Medicine 383.24 (2020), pp. 2302-2304. DOI: 10.1056/NEJMp2024046. eprint: https://doi.org/10. 1056/NEJMp2024046.

[8] Charlotte Jackson et al. "School Closures and Influenza: Systematic Review of Epidemiological Studies". In: BMJ Open 3.2 (2013). ISSN: 2044-6055. DOI: 10.1136/bmjopen-2012002149. eprint: https://bmjopen.bmj.com/content/3/2/e002149.full.pdf.

[9] Charlotte Jackson et al. "The Effects of School Closures on Influenza Outbreaks and Pandemics: Systematic Review of Simulation Studies". In: PLOS ONE 9.5 (May 2014), pp. 110. DOI: 10.1371/journal.pone.0097297.

[10] Nicholas G. Davies et al. "Age-Dependent Effects in the Transmission and Control of COVID-19 Epidemics". In: Nature Medicine (June 2020). ISSN: 1546-170X. DOI: 10.1038/ s41591-020-0962-9.

[11] Robert Verity et al. "Estimates of the Severity of Coronavirus Disease 2019: A Model-Based Analysis". In: The Lancet Infectious Diseases (2020).

[12] Joseph T Wu et al. "Estimating Clinical Severity of COVID-19 from the Transmission Dynamics in Wuhan, China". In: Nature medicine (2020), pp. 1-5.

[13] K Gaythorpe et al. Report 37: Children's Role in the COVID-19 Pandemic: A Systematic Review of Early Surveillance Data on Susceptibility, Severity, and Transmissibility. en. Tech. rep. Imperial College London, Nov. 2020.

[14] E Goldstein, M Lipsitch, and M Cevik. "On the Effect of Age on the Transmission of SARSCoV-2 in Households, Schools and the Community". In: The Journal of Infectious Diseases (Oct. 2020). ISSN: 0022-1899. DOI: 10.1093/infdis/jiaa691. eprint: https://academic.oup. com/jid/advance-article-pdf/doi/10.1093/infdis/jiaa691/34055049/jiaa691.pdf.

[15] Russell M. Viner et al. "Susceptibility to SARS-CoV-2 Infection Among Children and Adolescents Compared With Adults: A Systematic Review and Meta-Analysis". In: JAMA Pediatrics (Sept. 2020). ISSN: 2168-6203. DOI: 10.1001/jamapediatrics.2020.4573.

[16] Stefan Flasche and W John Edmunds. "The Role of Schools and School-Aged Children in SARS-CoV-2 Transmission". In: The Lancet Infectious Diseases (). ISSN: 1473-3099. DOI: 10.1016/S1473-3099(20)30927-0.

[17] Katherine A. Auger et al. "Association Between Statewide School Closure and COVID19 Incidence and Mortality in the US". In: JAMA 324.9 (Sept. 2020), pp. 859-870. ISSN: 0098-7484. DOI: 10.1001/jama.2020.14348.

[18] Seth Flaxman et al. "Estimating the Effects of Non-Pharmaceutical Interventions on COVID19 in Europe". In: Nature 584.7820 (Aug. 2020), pp. 257-261. ISSN: 1476-4687. DOI: 10. 1038/s41586-020-2405-7. 
[19] Chen Stein-Zamir et al. "A Large COVID-19 Outbreak in a High School 10 Days after Schools' Reopening, Israel, May 2020”. In: Eurosurveillance 25.29 (2020), p. 2001352.

[20] Sharif A Ismail et al. "SARS-CoV-2 Infection and Transmission in Educational Settings: A Prospective, Cross-Sectional Analysis of Infection Clusters and Outbreaks in England". In: The Lancet Infectious Diseases (). ISSN: 1473-3099. DOI: 10.1016/S1473-3099(20)30882-3.

[21] COVID-19 in Children and the Role of School Settings in Transmission - First Update. en. https://www.ecdc.europa.eu/en/publications-data/children-and-school-settings-covid19-transmission. Dec. 2020.

[22] Kanecia O Zimmerman et al. "Incidence and Secondary Transmission of SARS-CoV-2 Infections in Schools". In: Pediatrics (2021).

[23] Charlotte V Hobbs. "Factors Associated with Positive SARS-CoV-2 Test Results in Outpatient Health Facilities and Emergency Departments among Children and Adolescents Aged 18 Years-Mississippi, September-November 2020". In: MMWR. Morbidity and mortality weekly report 69 (2020).

[24] Seth Flaxman et al. "Report 13: Estimating the Number of Infections and the Impact of Non-Pharmaceutical Interventions on COVID-19 in 11 European Countries". In: (2020).

[25] Flávio C. Coelho et al. "Assessing the Spread of COVID-19 in Brazil: Mobility, Morbidity and Social Vulnerability". In: PLOS ONE 15.9 (Sept. 2020), pp. 1-11. DOI: 10.1371/journal. pone.0238214.

[26] Rosalind M. Eggo et al. "The Importance of Local Context in COVID-19 Models". In: Nature Computational Science 1.1 (Jan. 2021), pp. 6-8. ISSN: 2662-8457. DOI: 10.1038/ s43588-020-00014-7.

[27] Jennifer R Head et al. "The Effect of School Closures and Reopening Strategies on COVID19 Infection Dynamics in the San Francisco Bay Area: A Cross-Sectional Survey and Modeling Analysis". In: medRxiv (Jan. 2020), p. 2020.08.06.20169797. DOI: 10.1101/2020.08. 06.20169797.

[28] Timothy C. Germann et al. "Using an Agent-Based Model to Assess k-12 School Reopenings under Different COVID-19 Spread Scenarios - United States, School Year 2020/21". In: medRxiv (2020). DOI: 10.1101/2020.10.09.20208876. eprint: https://www.medrxiv.org/ content/early/2020/10/13/2020.10.09.20208876.full.pdf.

[29] Cohen, Jamie et al. "Maximizing Education While Minimizing COVID Risk: Priorities and Pitfalls for Reopening Schools". In: Institute for Disease Modeling (Aug. 2020).

[30] Guido Espana et al. "Impacts of K-12 School Reopening on the COVID-19 Epidemic in Indiana, USA". In: medRxiv (2020). DOI: 10.1101/2020.08.22.20179960. eprint: https: //www.medrxiv.org/content/early/2020/09/14/2020.08.22.20179960.full.pdf. 
[31] Jasmina Panovska-Griffiths et al. "Determining the Optimal Strategy for Reopening Schools, the Impact of Test and Trace Interventions, and the Risk of Occurrence of a Second COVID19 Epidemic Wave in the UK: A Modelling Study". English. In: The Lancet Child \&5 Adolescent Health 4.11 (Nov. 2020), pp. 817-827. ISSN: 2352-4642, 2352-4650. DOI: 10.1016/S23524642(20)30250-9.

[32] INS. "Seroprevalencia de SARS-CoV-2 durante la epidemia en Colombia: Estudio país". es. In: (Dec. 2020), p. 6.

[33] Michael A. Johansson et al. "SARS-CoV-2 Transmission From People Without COVID-19 Symptoms". In: JAMA Network Open 4.1 (Jan. 2021), e2035057-e2035057. ISSN: 2574-3805. DOI: 10.1001/jamanetworkopen.2020.35057.

[34] Lewis F. Buss et al. "Three-Quarters Attack Rate of SARS-CoV-2 in the Brazilian Amazon during a Largely Unmitigated Epidemic". In: Science 371.6526 (2021), pp. 288-292. ISSN: 0036-8075. DOI: 10.1126/science.abe9728. eprint: https://science.sciencemag.org/content/ 371/6526/288.full.pdf.

[35] Laura Di Domenico et al. "Modelling Safe Protocols for Reopening Schools during the COVID-19 Pandemic in France". In: medRxiv (2021). DOI: 10.1101/2020.05.08.20095521. eprint: https://www.medrxiv.org/content/early/2021/01/14/2020.05.08.20095521.full. pdf.

[36] Ryan S. McGee et al. "Model-Driven Mitigation Measures for Reopening Schools during the COVID-19 Pandemic". In: medRxiv (2021). DOI: 10.1101/2021.01.22.21250282. eprint: https://www.medrxiv.org/content/early/2021/01/26/2021.01.22.21250282.full.pdf.

[37] Pirous Fateh-Moghadam et al. "Contact Tracing during Phase I of the COVID-19 Pandemic in the Province of Trento, Italy: Key Findings and Recommendations". In: medRxiv (2020). DOI: $10.1101 / 2020.07 .16 .20127357$.

[38] Taylor Heald-Sargent et al. "Age-Related Differences in Nasopharyngeal Severe Acute Respiratory Syndrome Coronavirus 2 (SARS-CoV-2) Levels in Patients with Mild to Moderate Coronavirus Disease 2019 (COVID-19)". In: JAMA Pediatrics (July 2020). ISSN: 2168-6203. DOI: $10.1001 /$ jamapediatrics.2020.3651.

[39] "Minesota Population Center". "Integrated Public Use Microdata Series: Version 6.5[Dataset]". In: (2017).

[40] Secretaría Distrital de Planeación. http://www.sdp.gov.co/\#enlace-de-interes.

[41] COLOMBIA - Censo Nacional de Población y Vivienda - CNPV - 2018. http://microdatos.dane.gov.co/inde

[42] Oferta Institucional Personas Mayores - Todas Las Ofertas. https://www.minsalud.gov.co/proteccionsocial/] 1D1E-4A3B-9973-FE97E04FBD27\%7D.

[43] Instituciones de Educación Superior - Ministerio de Educación Nacional de Colombia. https://www.mineducacion.gov.co/1759/w3-article-231240.html?_noredirect=1.

[44] COVID-19 Community Mobility Report. https://www.google.com/covid19/mobility?hl=en.

[45] Impacto de Las Políticas de Aislamiento a Partir Del COVID 19. en. https://covid.grandata.com. 
[46] Google Trends. en-US. https://trends.google.com/trends/?geo=US.

[47] Coronavirus Colombia. https://www.ins.gov.co/Noticias/Paginas/Coronavirus.aspx.

[48] John J Grefenstette et al. "FRED (A Framework for Reconstructing Epidemic Dynamics): An Open-Source Software System for Modeling Infectious Diseases and Control Strategies Using Census-Based Populations". In: BMC public health 13.1 (2013), p. 940.

[49] Evan L Ray et al. "Ensemble Forecasts of Coronavirus Disease 2019 (COVID-19) in the U.S." In: medRxiv (2020). DOI: 10.1101/2020.08.19.20177493. eprint: https://www . medrxiv.org/content/early/2020/08/22/2020.08.19.20177493.full.pdf.

[50] Katriona Shea et al. "COVID-19 Reopening Strategies at the County Level in the Face of Uncertainty: Multiple Models for Outbreak Decision Support". In: medRxiv (2020). DOI: 10.1101/2020.11.03.20225409. eprint: https://www.medrxiv.org/content/early/2020/11/ 05/2020.11.03.20225409.full.pdf.

[51] Tapiwa Ganyani et al. "Estimating the Generation Interval for COVID-19 Based on Symptom Onset Data". en. In: medRxiv (Mar. 2020), p. 2020.03.05.20031815. ISSN: 10.1101/2020.03.05.20031815. DOI: $10.1101 / 2020.03 .05 .20031815$.

[52] N Brazeau et al. "Report 34: COVID-19 Infection Fatality Ratio: Estimates from Seroprevalence". In: (2020).

[53] Derek K Chu et al. "Physical Distancing, Face Masks, and Eye Protection to Prevent Person-to-Person Transmission of SARS-CoV-2 and COVID-19: A Systematic Review and Meta-Analysis". In: The Lancet (2020).

[54] Matthias Templ et al. "Simulation of Synthetic Complex Data: The R Package simPop". In: Journal of Statistical Software 79.10 (2017), pp. 1-38. DOI: 10.18637/jss.v079.i10.

[55] T. Alex Perkins et al. "Estimating Unobserved SARS-CoV-2 Infections in the United States". In: Proceedings of the National Academy of Sciences (2020). ISSN: 0027-8424. DOI: 10.1073 /pnas.2005476117.

[56] Aaron A. King et al. pomp: Statistical Inference for Partially Observed Markov Processes. Manual. 2020.

[57] Aaron A. King, Dao Nguyen, and Edward L. Ionides. "Statistical Inference for Partially Observed Markov Processes via the R Package pomp". In: Journal of Statistical Software 69.12 (2016), pp. 1-43. DOI: 10.18637/jss.v069.i12.

[58] Lauren M. Kucirka et al. "Variation in False-Negative Rate of Reverse Transcriptase Polymerase Chain Reaction-Based SARS-CoV-2 Tests by Time Since Exposure". In: Annals of Internal Medicine 173.4 (May 2020), pp. 262-267. ISSN: 0003-4819. DOI: 10.7326/M20-1495.

[59] Janko van Beek et al. "From More Testing to Smart Testing: Data-Guided SARS-CoV-2 Testing Choices". In: medRxiv (2020). DOI: 10.1101/2020.10.13.20211524. eprint: https: //www.medrxiv.org/content/early/2020/10/14/2020.10.13.20211524.1.full.pdf.

[60] OPHI. Global Multidimensional Poverty Index 2018: The Most Detailed Picture to Date of the World's Poorest People / OPHI. https://ophi.org.uk/global-multidimensional-povertyindex-2018-the-most-detailed-picture-to-date-of-the-worlds-poorest-people/. 


\section{Supplementary material}
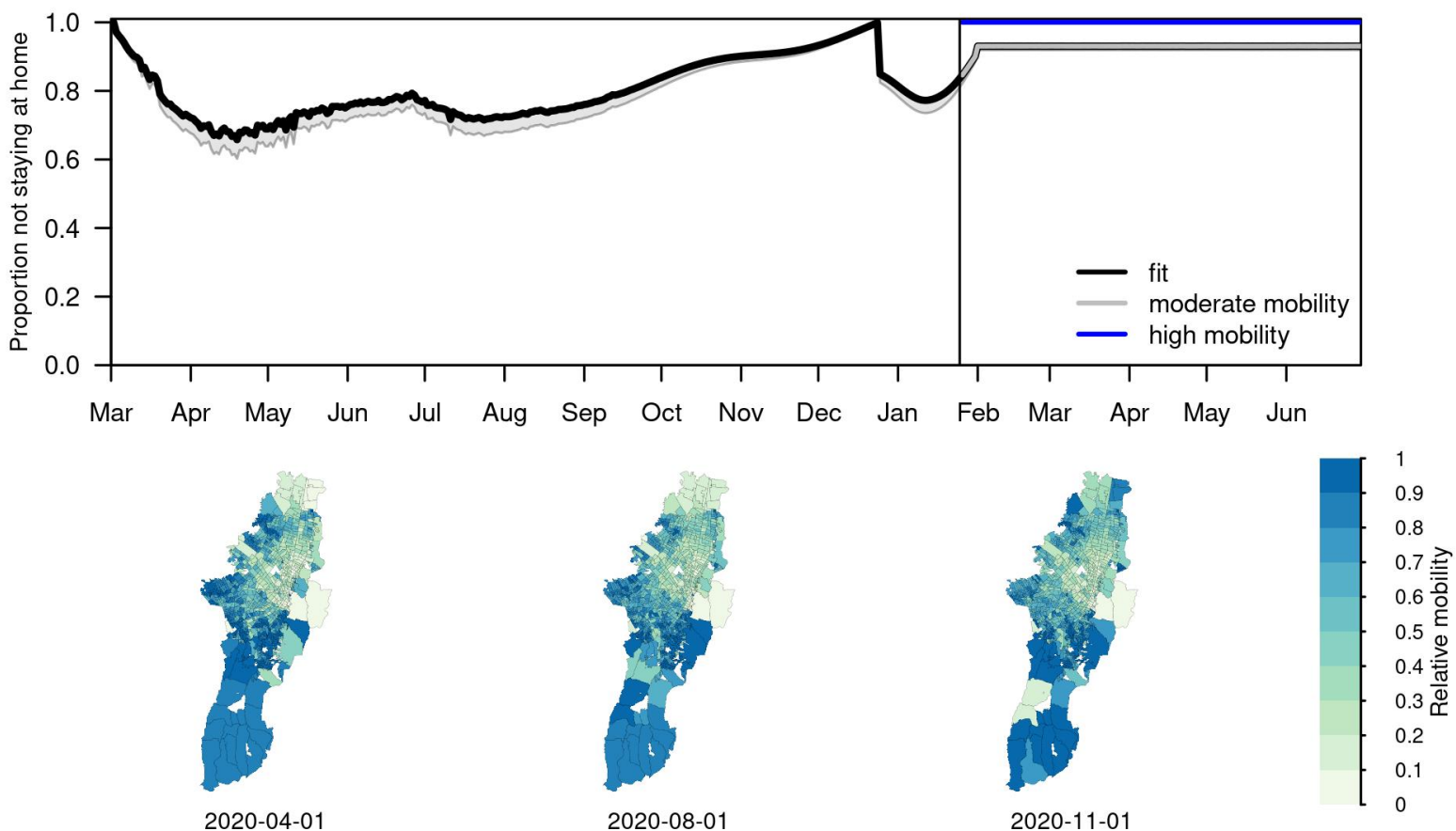

Figure S1. Adjusted mobility in time and space for the city of Bogotá. Top panel shows the calibrated reduction in mobility over time. Bottom panel shows the geographical distribution of mobility restrictions at three different time points. Darker blue colors represent less mobility, and lighter green colors represent more mobility. 

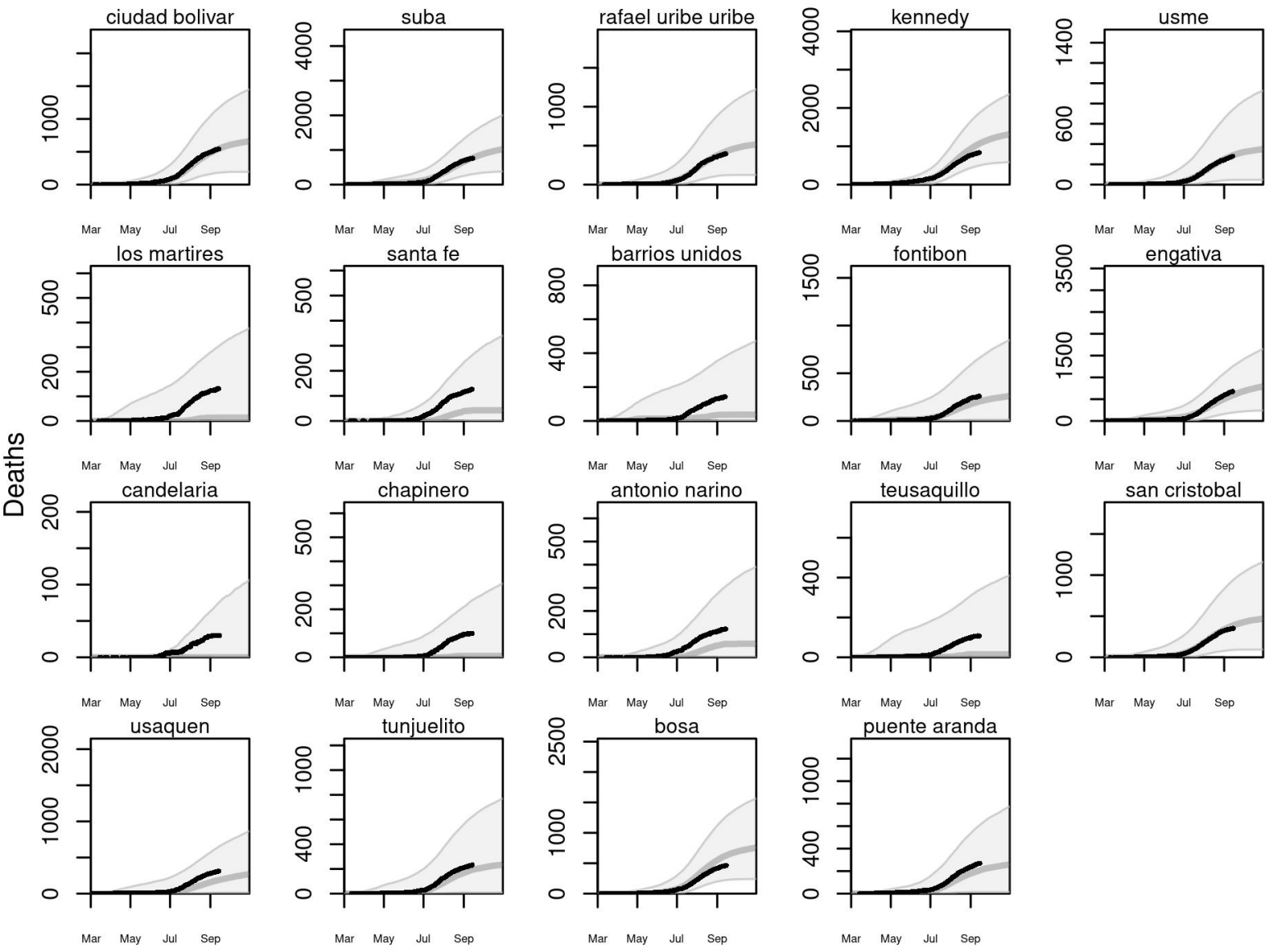

Mar May Jul Sop

Mar May Jul Sop

Mar May Jul Sep
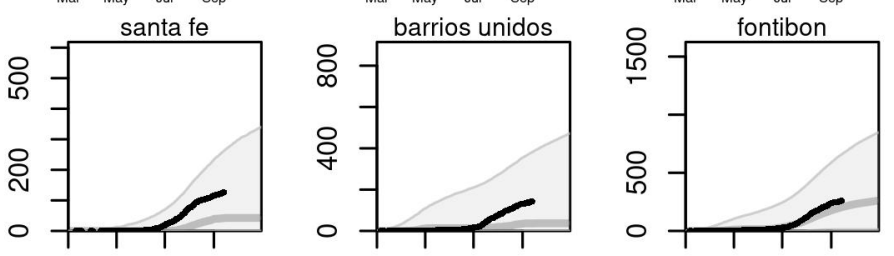

Mar May Jul Sop

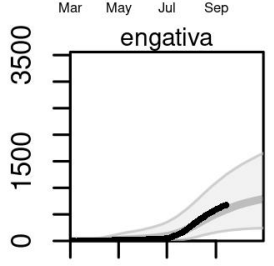

Mar May Jul Sep

chapinero

Mar May Jul Sep

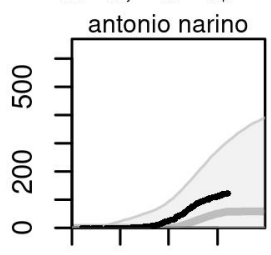

Mar May Jul Sep

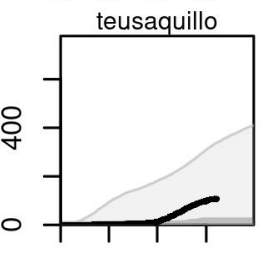

Mar May Jul Sep

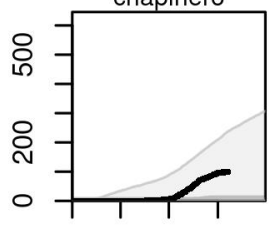

Mar May Jul Sep

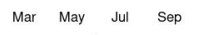

Mar May Jul Sep
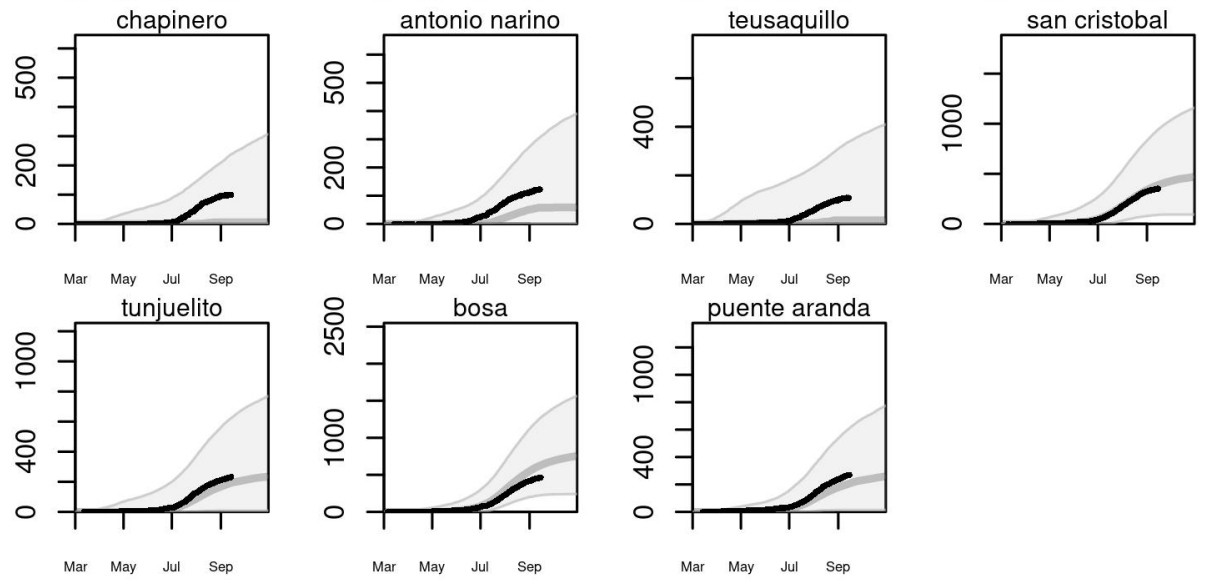

Figure S2. Model comparison with data excluded from calibration for each locality. Each panel shows the daily cumulative incidence of deaths for each locality in Bogotá, Colombia. Black line represents the official data, and gray lines and shaded areas represent the model median estimates and 95\% CrI. Assumption of lower (50\%) susceptibility in $<10$ years. 

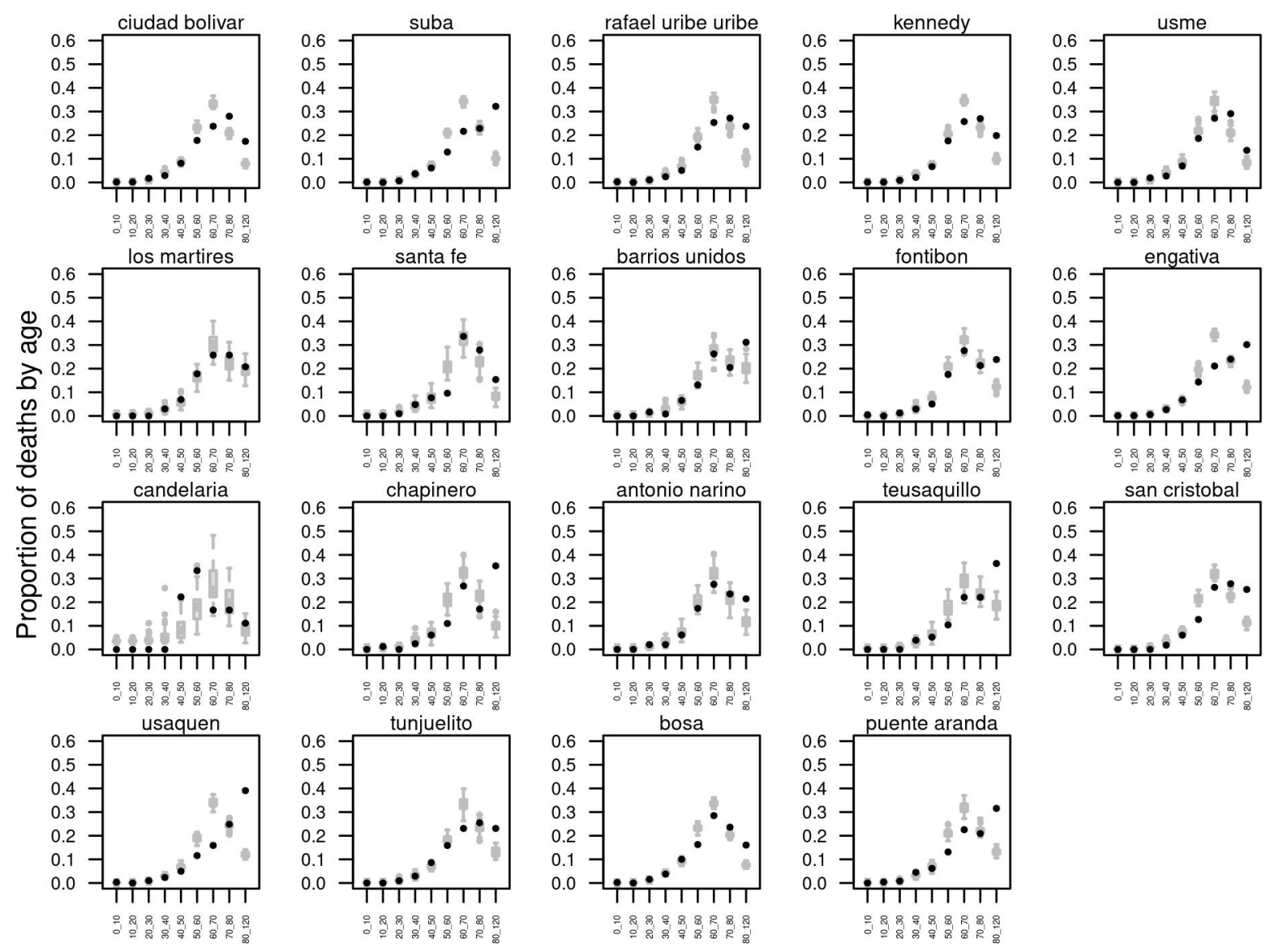

Age group

Figure S3. Model comparison with age-specific data excluded from calibration for each locality. Each panel shows the proportion of total deaths by each age group in each locality in Bogotá, Colombia. Black points show the data and gray box plots show the model estimates. Assumption of lower (50\%) susceptibility in $<10$ years. 


\section{Estimated attack rate in Bogota}

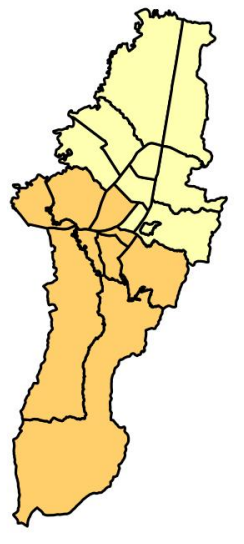

2020-07-07

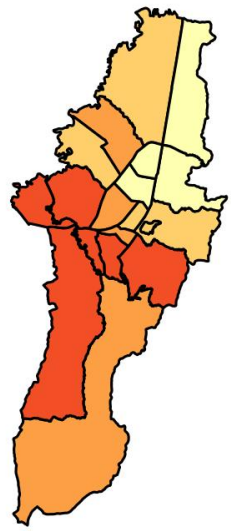

2020-09-07

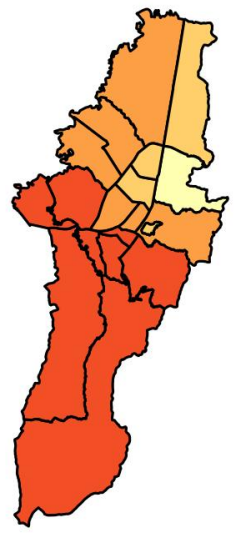

2020-11-07

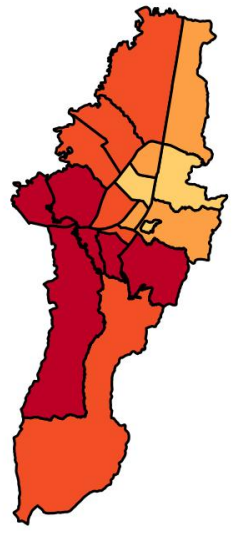

2021-01-07

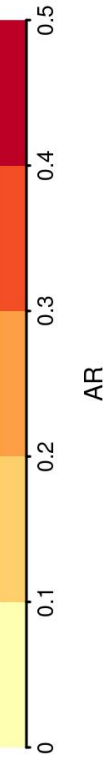

Figure S4. Model estimates of attack rate by locality in Bogotá at different times points. Darker red colors represent a higher proportion of the population infected by each date, and lighter yellow colors represent a lower proportion of the population infected by each date. Assumption of lower $(50 \%)$ susceptibility in $<10$ years. 


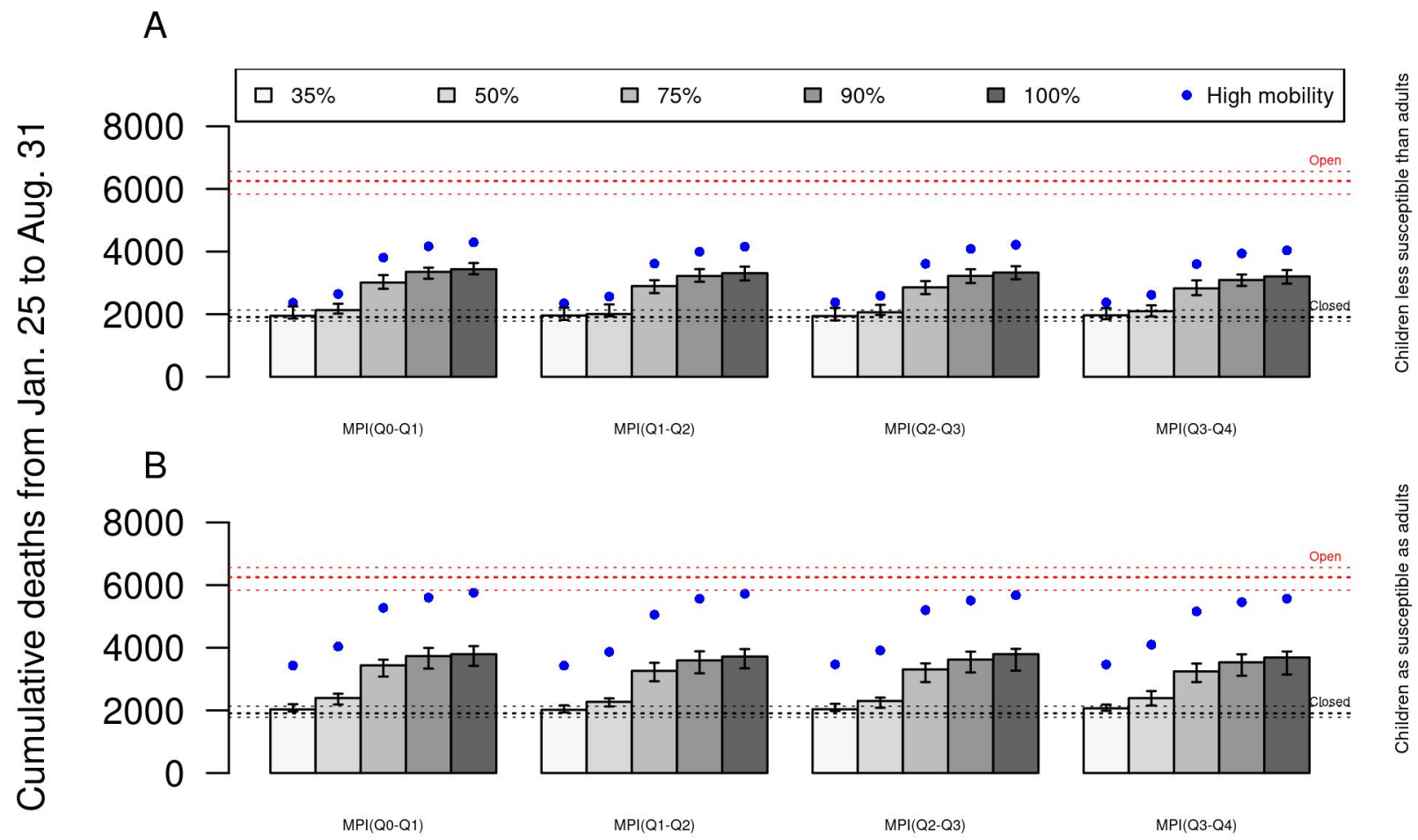

Figure S5. Total cumulative deaths under different school reopening strategies from January 25 to August 31, 2021. Cumulative deaths of scenarios in which schools reopen by multidimensional poverty index of schools. A) Assumption of lower susceptibility for $<10$ years. B) Assumption of equal susceptibility to infection for all ages. In all scenarios, red dotted lines show the median and 95\% CrI of the scenario with full schools reopening (with face masks), and the black dotted line shows the median and $95 \% \mathrm{CrI}$ of the scenario with all schools closed. Blue dots show a scenario with high mobility when schools reopen. In all scenarios, we assumed long-term protection after SARS-CoV-2 infection. 


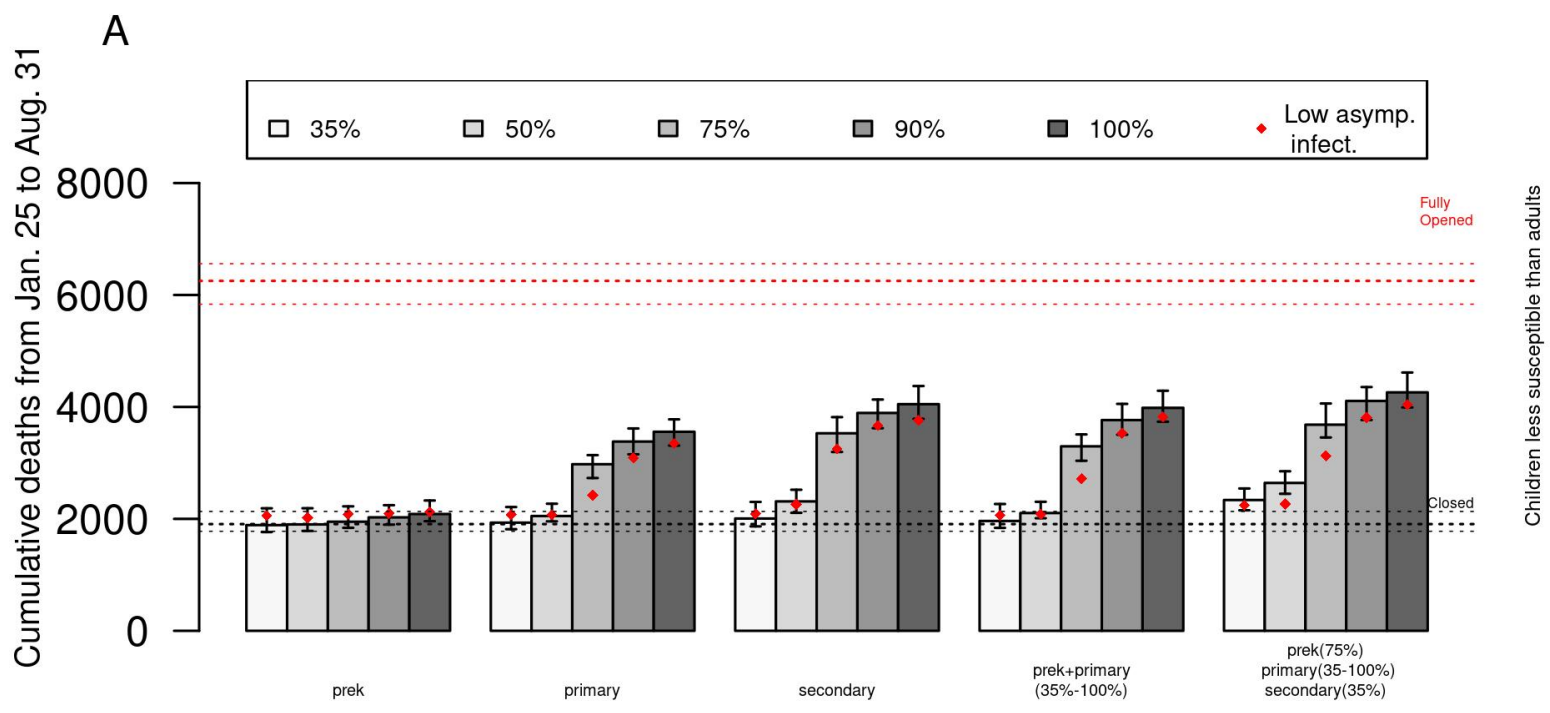

Figure S6. The impact of lower asymptomatic infectiousness in the total cumulative deaths under different school reopening strategies from January 25 to August 31, 2021. A) Cumulative deaths of scenarios in which schools reopen by grades with an assumption of lower (50\%) susceptibility in $<10$ years. From left to right, the first group of bars show exclusive reopening by grade groups in which the other grades remain closed. The fourth group of bars (prek+primary) represents a scenario in which prek primary and primary reopen at different capacities but secondary remains closed. The last group shows a scenario in which all grades go to in-person school at some level, with prek fixed at $75 \%$, secondary fixed at $35 \%$, and primary varying from $35 \%$ to $100 \%$. Red diamonds show the median estimate of the same scenario with $75 \%$ infectiousness of asymptomatics. In all scenarios, we assumed long-term protection after SARSCoV-2 infection and lower (50\%) susceptibility in $<10$ years. 
A
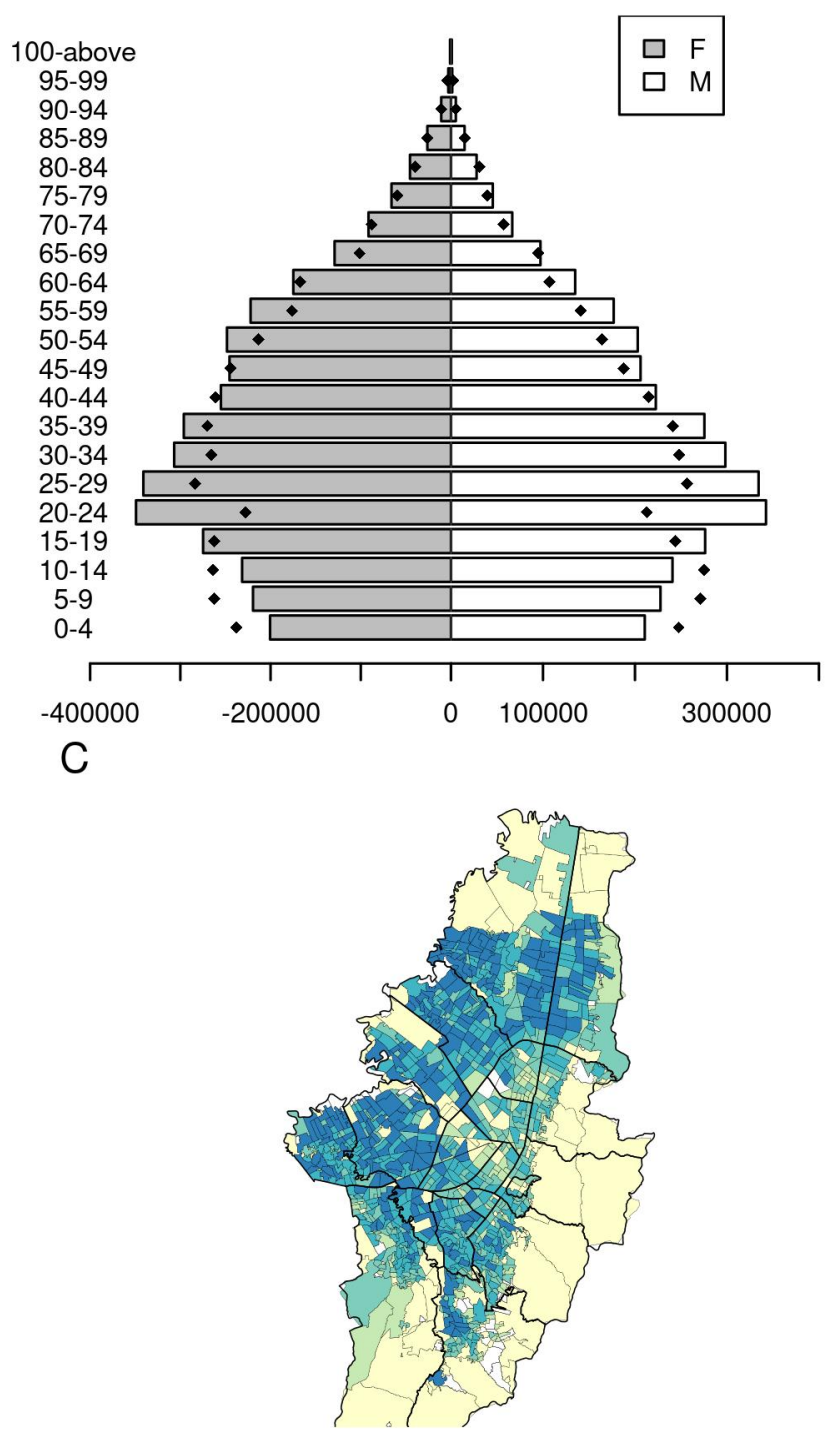
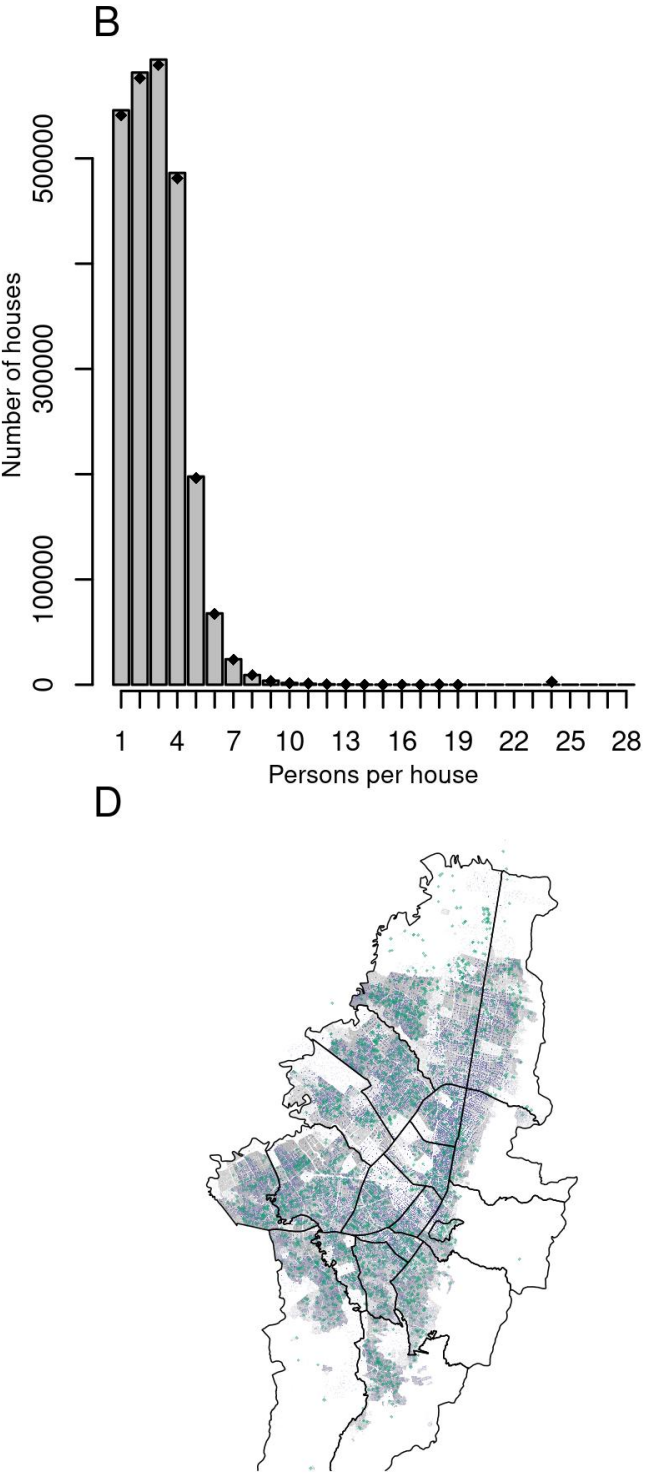

Figure S7. Synthetic population of Bogotá, Colombia. A) Comparison of aggregated population by age and sex. Bars show the synthetic population and black dots the census data from Bogotá, Colombia. B) Household distribution for the synthetic population (bars) and data(points). C) Population density by Census Tract (Unidad Catastral). Darker colors show higher population density. D) Location of households (gray), schools (green), and workplaces (purple) in the synthetic population of Bogotá. 


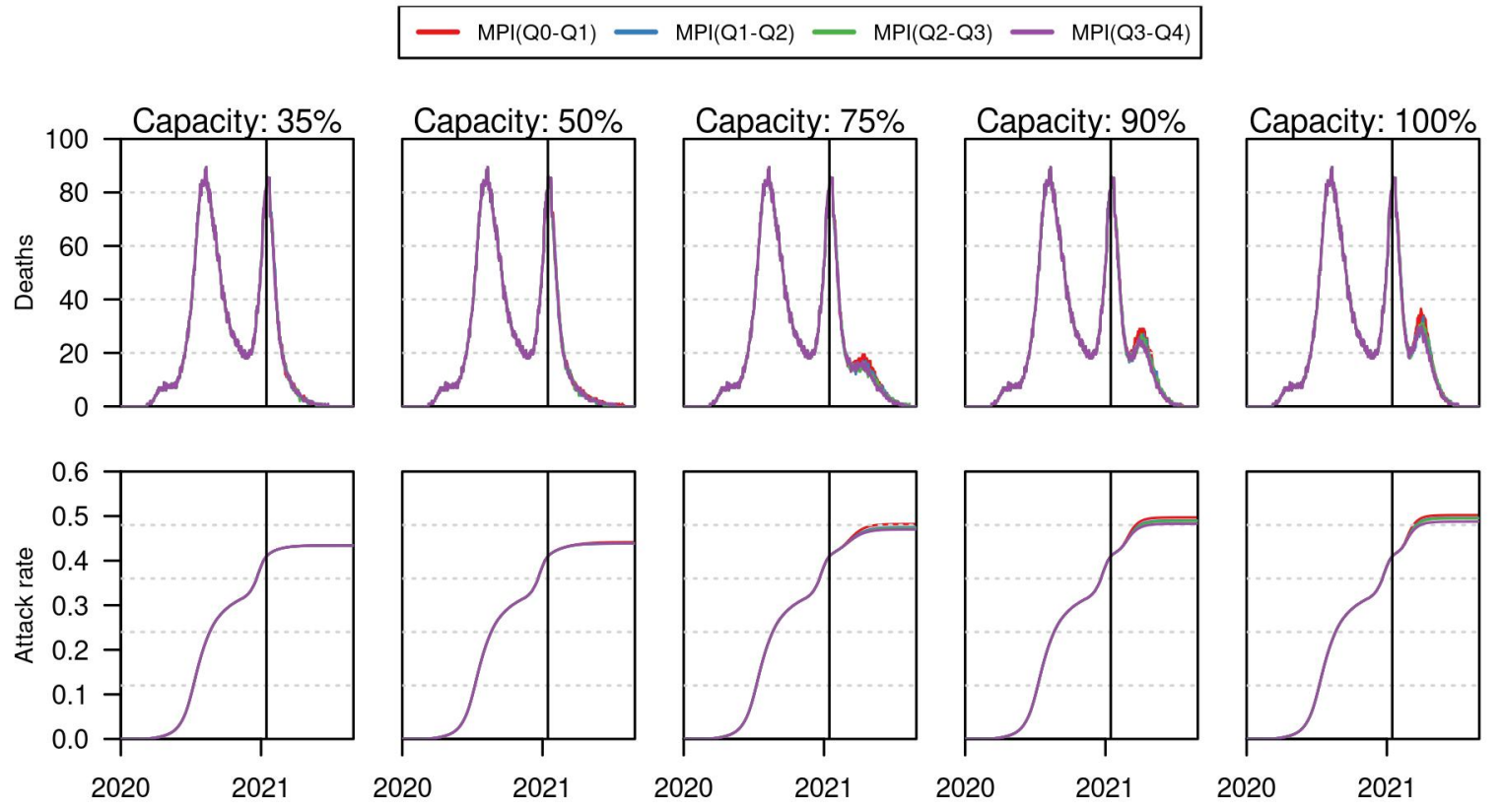

Figure S8. The impact of delaying school reopening by multidimensional poverty index of schools. We assumed lower $(50 \%)$ susceptibility in $<10$ years. Each column shows a different capacity level. Each group of MPI shows a set of schools grouped according to their MPI. Bottom panel shows the median estimate of attack rates for each scenario. Vertical black line shows the initial date of school reopening (January 25, 2021). All scenarios were simulated until August 31, 2021. 

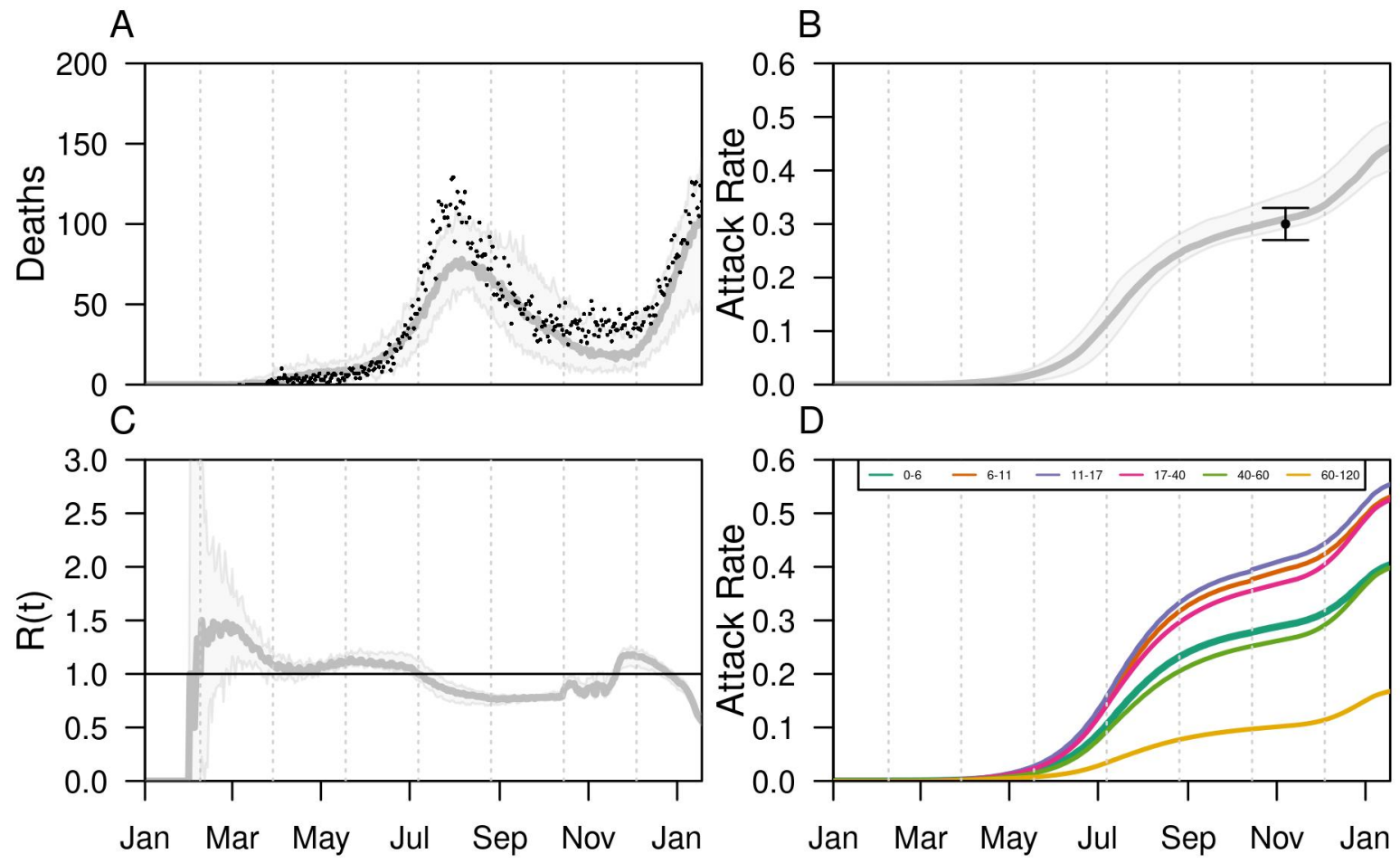

Figure S9. Model fit to data with assumption of equal susceptibility for all ages. A) Model fit to daily incidence of deaths. Black dots show the official data, and gray lines show the median estimate of the model with the $95 \% \mathrm{CrI}$ represented by gray-shaded curves. B) Model estimates of attack rate in time represented by gray line (median) and shaded area (95\% CrI). The point and arrows show the median estimates and CI of official serological study in Bogotá. C) Estimated reproduction number in time. D) Estimated attack rate in time for different age groups. 

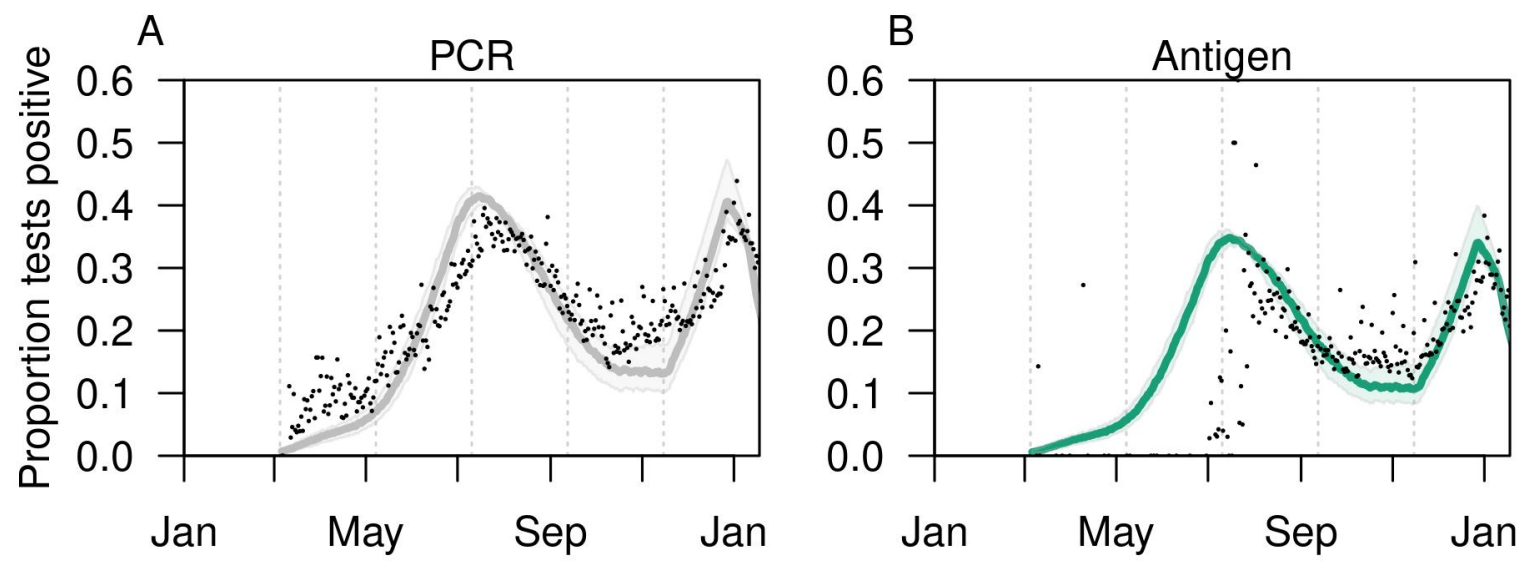

Figure S10. Model comparison to test positive data. We assumed lower (50\%) susceptibility in $<10$ years. A) daily proportion of PCR tests positive by date of sample. Gray line and shaded area show the median and $95 \% \mathrm{CrI}$ of the model simulations. Black points show the data. B) daily proportion of antigen tests positive by date of sample. Gray line and shaded area show the median and $95 \% \mathrm{CrI}$ of the model simulations. Black points show the data.

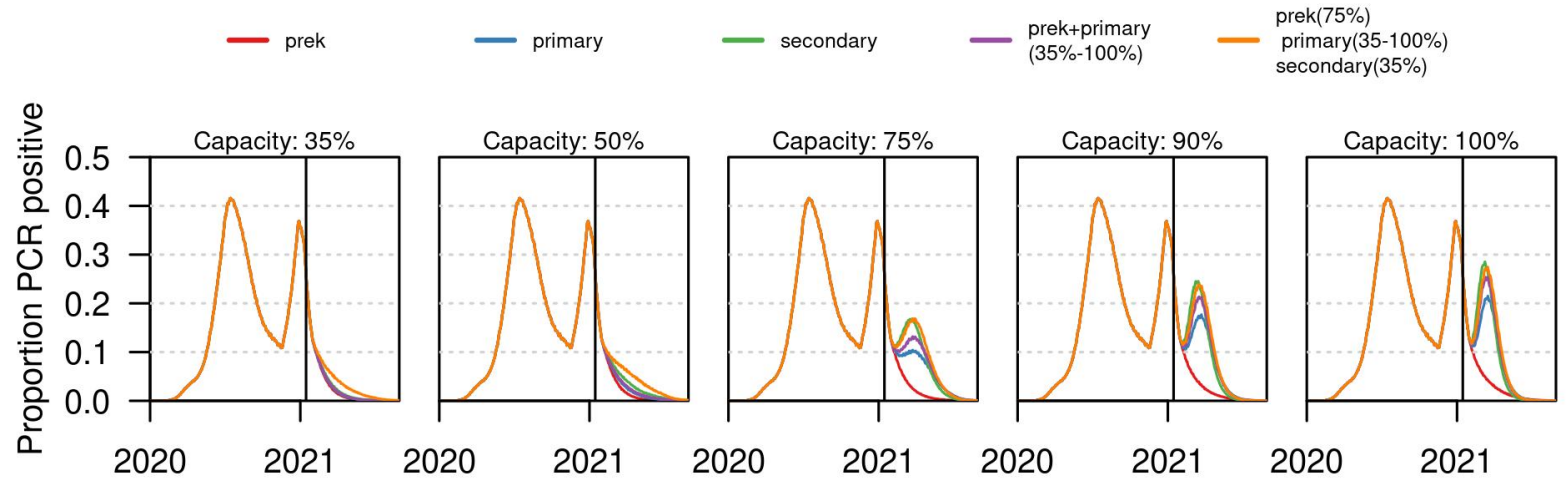

Figure S11. The impact of school reopening on the positive rate of PCR testing for different grades in in-person school. Assumption of lower (50\%) susceptibility in $<10$ years. Each column shows a different capacity, and each line shows a different strategy of school reopening base on grades. Black vertical line shows the baseline date of school reopening. 


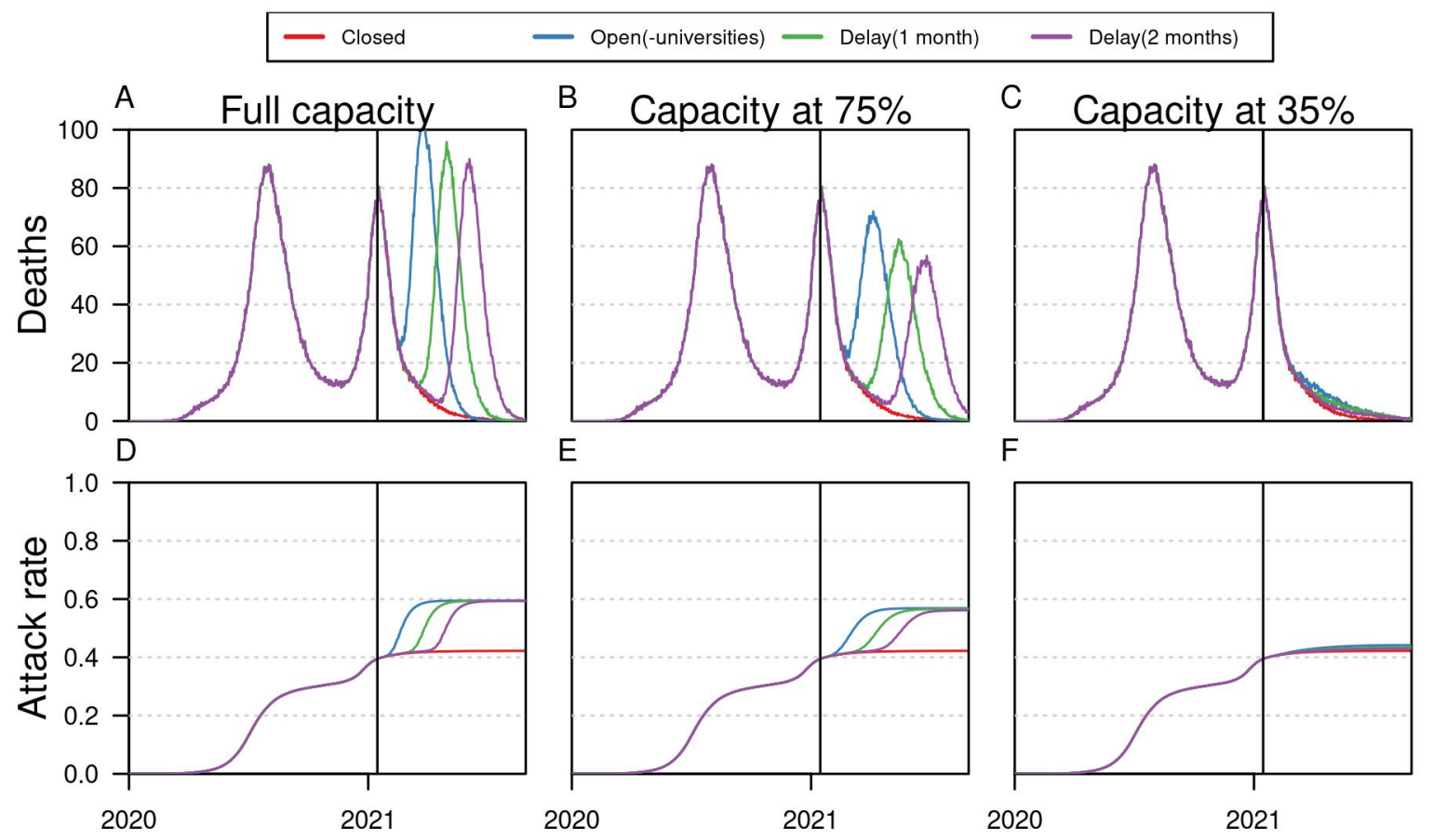

Figure S12. The impact of delaying school reopening. Each column shows a different capacity level. Red lines represent a scenario in which all schools remain closed, blue lines represent K-12 schools open, green and purple lines show scenarios of delaying school reopening by 1 and 2 months, respectively. Top panel shows the median estimate of daily incidence of deaths. Bottom panel shows the median estimate of attack rates for each scenario. Vertical black line shows the initial date of school reopening (January 25, 2021). In these scenarios, we assumed equal susceptibility for all ages. 